\title{
THE BROADWELL MODEL IN A THIN CHANNEL *
}

\author{
ANDREW J. CHRISTLIEB, ${ }^{\dagger}$, JAMES A. ROSSMANITH, ${ }^{\ddagger}$, AND PETER SMEREKA $\S$
}

\begin{abstract}
In this paper we are concerned with the limiting behavior of gas flow in a thin channel as described by the Broadwell model. The Broadwell model is a simplified kinetic description for gas dynamics where the main assumption is that the particle distribution function can be represented by a discrete number of velocities. Starting from the Broadwell model and the appropriate boundary conditions we derive two 1D models for gas transport in a thin channel. In the limit of no interparticle collisions the 1D model is the well known telegraph equation. In the case of collisional flow the $1 \mathrm{D}$ model is a system of three first-order hyperbolic PDEs. Both 1D models are validated through numerical simulations that compare the $1 \mathrm{D}$ models to the $2 \mathrm{D}$ Broadwell system. Furthermore, in the limit of no inter-particle collisions we are able to rigorously show that under a diffusive scaling the solutions of the full Broadwell model converge weakly to solutions of the diffusion equation. Under a hyperbolic scaling we are able to show that solutions to the collisionless Broadwell model converge weakly to the solutions of the telegraph equation. Finally, we derive a long-time asymptotic formula for the solution of the collisionless Broadwell system, which reveals oscillations that explain why the convergence in the diffusive and hyperbolic scalings must be weak. Due to the nonlinearity of the inter-particle collisions, we are not able to prove rigorous convergence results for the collisional Broadwell system.
\end{abstract}

Key words. Discrete velocity models, Broadwell model, Thin channel, Telegraph equation, Kinetic modeling, Rarefied gas dynamics.

AMS subject classifications. 76P05, 82B40, 35L50, 35K10

\section{Introduction}

Recent advances in the manufacturing of microscale technology have made it possible to construct mechanical systems on the micro-scale [19]. It was quickly discovered that systems on the scale of tens of microns can behave quite differently from their macroscale counterparts. The effects of decreasing the gas density have been discussed in review articles in the MEMS community [20, 22] and are discussed in standard texts on gas dynamics $[8,14,31]$. In addition, experiments have shown that even mildly rarefied gas flows behave far different on the microscale then they do on the macroscale $[15,27,33,34]$. This initial work has generated a great deal of interest in trying to describe the fundamental physics for systems on this scale with the goal of better predicting the behavior of these systems.

A subclass of these devices (e.g., micro-sensors and/or micro-pumps) have micro/nano scale flow channels cut in them. The channel width in these devices may range from tens of microns down to tens of nanometers [28, 29]. Because these devices are often intended to be operated at atmospheric pressure where the mean distance a particle travels before undergoing a binary collision with another particle is about 70 nanometers in air, the gas may appear very dilute on the scale of these devices.

*Received: April 12, 2004; accepted (in revised version): August 9, 2004. Communicated by Pierre Degond.

${ }^{\dagger}$ Department of Mathematics, University of Michigan, 525 East University Ave., Ann Arbor, MI 48109-1109, USA (christli@umich.edu).

${ }^{\ddagger}$ Department of Mathematics, University of Michigan, 525 East University Ave., Ann Arbor, MI 48109-1109, USA (rossmani@umich.edu).

$\S$ Department of Mathematics, University of Michigan, 525 East University Ave., Ann Arbor, MI 48109-1109, USA (psmereka@umich.edu). 
The dynamics of a gas can be modelled using the Boltzmann equation:

$$
\frac{\partial f}{\partial t}+\vec{u} \cdot \nabla f=\left\{\frac{\delta f}{\delta t}\right\}_{c}
$$

where $f=f(\vec{x}, \vec{u}, t)$ is the particle distribution function in phase-space. The left-hand side of this equation represents particle transport while the right-hand side models binary collisions. A measure of the diluteness of a gas is the Knudsen number, $K_{n}$, which is the ratio of the mean distance between inter-particle collisions, $\lambda$, and the characteristic length scale of the system, $L$. The relative importance of the collision operator is directly tied to the Knudsen number, $K_{n}$. If the Knudsen number is small then the flow is dominated by binary collisions. In this case, the solution of the Boltzmann equation can be approximated by a function of the type:

$$
f_{M}=\rho(\vec{x}, t) \exp \left\{-\frac{\left(u_{0}-u(\vec{x}, t)\right)^{2}}{T(\vec{x}, t)}\right\}
$$

where $f_{M}$ is called a local Maxwellian. In this limit one can employ the ChapmanEnskog formalism to derive transport equations for the density, $\rho(\vec{x}, t)$, velocity, $u(\vec{x}, t)$, and temperature, $T(\vec{x}, t)[14]$. However, when $K_{n}$ is not small this approximation to the Boltzmann equation fails.

For the micro/nano devices, the Knudsen number is typically in the range $0.01 \leq$ $K_{n} \leq 10$. As the width of the channel reduces to the point where $K_{n}>1$, the gas enters the free molecular flow regime in which the gas particles are more likely to have a collision with the walls of the system than with each other. This regime has been studied analytically as one possible limit of the Boltzmann equation by Babovsky [2,3] and Börgers et al. [6]. These articles consider the long-time behavior of the Boltzmann equation in the limit of no particle-particle collisions; this limit is sometimes referred to as the Knudsen gas limit. Babovsky [2] describes the long-time evolution of a Knudsen gas in a thin tube. Börgers et al. [3, 6] and Babovsky [2] discuss the behavior of a Knudsen gas for flow in a thin channel. In these articles, it is shown that under an appropriate scaling the long-time behavior of the Knudsen gas converges to solutions of the diffusion equation. In addition, Börgers et al. [6] have shown that the time scale on which diffusion takes place is sensitive to the model used to describe the distribution of particles reflecting from the wall. The approach taken in these articles is based on statistical arguments regarding the behavior of small angle collisions with the wall of the channel or tube. In addition, Golse [21], Dogbé [18], and Boatto and Golse [9] develop non-statistical arguments to show that solutions of the Knudsen gas for thin channel flow have a diffusion limit as the depth of the channel goes to zero. Degond and Mancini [17] and Degond, Latocha, Mancini, and Mellet [16] rigorously showed that a diffusion limit is also reached by an electron gas in both the collisionless and collisional cases, respectively. Finally, Aoki and Degond [1] showed that a Knudsen gas that is driven by temperature gradients along the channel walls can be approximated by a convection-diffusion equation.

In this work, we consider the dynamics of a discrete velocity model in a thin channel. Because the model we consider is a significant simplification of the full Boltzmann equation, we are able to extract more detailed information than previous work on the Knudsen gas in a thin channel. The major simplifying assumption of the model is that the gas particles can only travel along a discrete number of directions. Broadwell $[10,11]$ developed the first discrete velocity model as a tool for understanding 
shock formation in a gas. The success of this simple model inspired a great deal of work on discrete velocity models. Caflisch $[12,13]$ studied the fluid dynamic limit of the Broadwell model and was able to show that solutions of the 1D Broadwell model converge weakly to solutions of the fluid dynamic equations. The literature on discrete velocity models has become quite extensive. The review article by Platkowski and Illner [26] discusses much of the know theory about the fluid dynamic limit and the numerical schemes derived for fluid dynamics based on discrete velocity models. More recent work has involved using discrete velocity models to study rarefied gas dynamics. Valougeorgis and Naris [30] used discrete velocity models in the simulation of high $K_{n}$ gas flows in a microchannel. Bellouquid [7] has shown that under the right scaling, solutions of the collisional Broadwell model in the infinite domain converge to solutions of a wave equation.

The paper is broken into two main parts. In the first part, we derive depthaveraged models for flow in a thin channel for both the collisionless and collisional Broadwell model. The collisionless equations lead to a telegraph equation, while the collisional equations lead to a system of three first-order hyperbolic PDEs. In the second part, we show that under a hyperbolic scaling the solutions of the Broadwell model converge to solutions of our 1D model in the weak sense. Under a diffusive scaling, we show that the collisionless Broadwell model converges weakly to solutions of a diffusion equation. Finally, we derive a long-time asymptotic formula for the solution of the collisionless Broadwell system, which reveals oscillations that explain why the convergence in the diffusive and hyperbolic scalings must be weak.

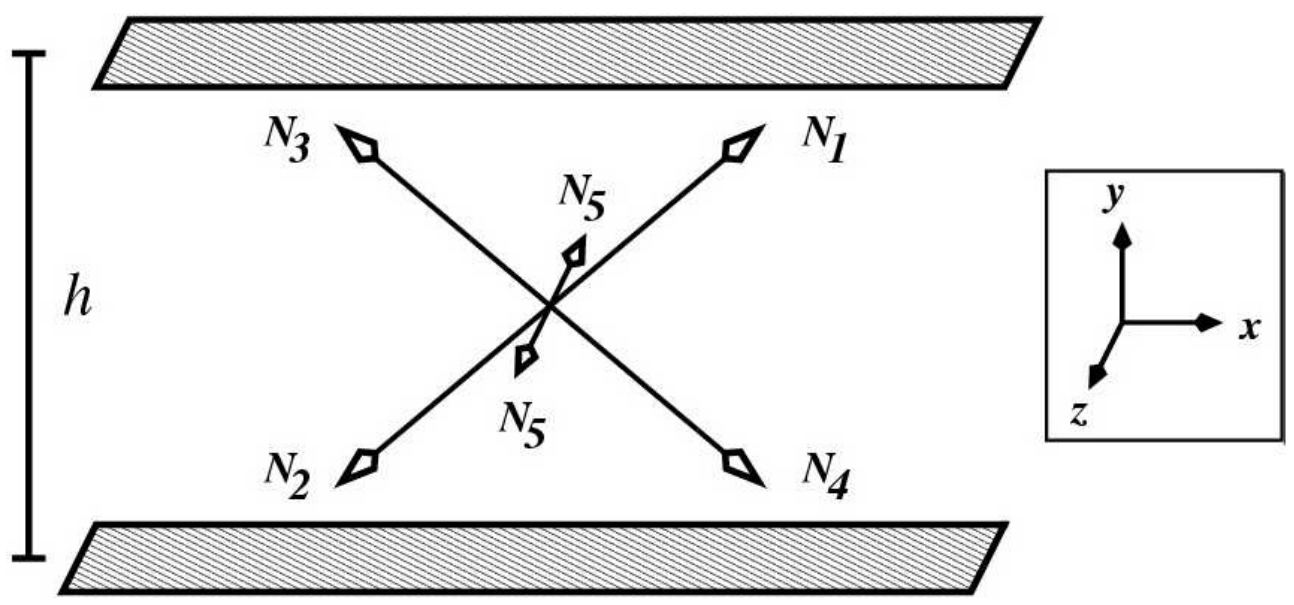

FIG. 1.1. The Broadwell model in a thin channel and no variation in the $z$-direction. Without collisions $(\sigma=0)$ only $N_{1}, N_{2}, N_{3}$, and $N_{4}$ are dynamically important. With collisions $(\sigma \neq 0)$ an additional component, $N_{5}$, is introduced to denote the particles that end up travelling in the $z$ direction after collisions. In the collisional case we do not need to consider two densities in the (+) and (-) z-directions (e.g., $N_{5}$ and $N_{6}$ ) since $N_{5} \equiv N_{6}$ in order to maintain zero momentum in the $z$-direction.

\section{Derivation of a collisionless thin-channel model}

We consider a version of the 6-velocity model originally proposed by Broadwell as a discrete velocity approximation to the Boltzmann equations [10]. In Broadwell's model, all particles move at a constant speed $c$ and are allowed only to travel in six directions: $\pm x, \pm y$, and $\pm z$. We will study this model in an infinitely long channel 
of height $H$. We consider only solutions that are independent of $z$, and we rotate Broadwell's model by $45^{\circ}$ degrees so that particles that are incident on the wall are reflected down the channel (see Figure 1.1). We note that in order to obtain 2D solutions with zero $z$-momentum it is required that the number of particles in the $(+)$ and $(-) z$-directions are equal for all $(x, y, t)$. Therefore, the system considered in this work is described entirely by five directions: $N_{1}, N_{2}, N_{3}, N_{4}$, and $N_{5}$.

We first consider the completely collisionless case. This further reduces the original 6-velocity model to a 4 -velocity model $\left(N_{1}-N_{4}\right)$, because in the absence of collisions we can assert without loss of generality that $N_{5} \equiv 0$ for all $(x, y, t)$. After non-dimensionalization, the collisionless 4-velocity Broadwell model for flow in a narrow channel that we consider in this work is given by the following four advection equations:

$$
\begin{aligned}
& \frac{\partial N_{1}}{\partial t}+\frac{\partial N_{1}}{\partial x}+\frac{\partial N_{1}}{\partial y}=0, \\
& \frac{\partial N_{2}}{\partial t}-\frac{\partial N_{2}}{\partial x}-\frac{\partial N_{2}}{\partial y}=0, \\
& \frac{\partial N_{3}}{\partial t}-\frac{\partial N_{3}}{\partial x}+\frac{\partial N_{3}}{\partial y}=0, \\
& \frac{\partial N_{4}}{\partial t}+\frac{\partial N_{4}}{\partial x}-\frac{\partial N_{4}}{\partial y}=0,
\end{aligned}
$$

on $(x, y) \in[-\infty, \infty] \times[0, h]$, where $h$ is the non-dimensional channel depth. Nondimensional parameters are related to dimensional quantities in the following way:

$$
N^{\star}=L^{-3} N, \quad x^{\star}=L x, \quad y^{\star}=L y, \quad t^{\star}=(L / c) t, \quad \text { and } \quad h=H / L,
$$

where $L$ is the characteristic length scale of the initial condition. Coupling between the four velocities comes from interaction at the top and bottom walls of the channel:

$$
\begin{aligned}
& \left.N_{1}\right|_{y=0}=\left.(1-\alpha) N_{4}\right|_{y=0}+\frac{\alpha}{2}\left(\left.N_{2}\right|_{y=0}+\left.N_{4}\right|_{y=0}\right) \\
& \left.N_{3}\right|_{y=0}=\left.(1-\alpha) N_{2}\right|_{y=0}+\frac{\alpha}{2}\left(\left.N_{2}\right|_{y=0}+\left.N_{4}\right|_{y=0}\right) \\
& \left.N_{2}\right|_{y=h}=\left.(1-\alpha) N_{3}\right|_{y=h}+\frac{\alpha}{2}\left(\left.N_{1}\right|_{y=h}+\left.N_{3}\right|_{y=h}\right) \\
& \left.N_{4}\right|_{y=h}=\left.(1-\alpha) N_{1}\right|_{y=h}+\frac{\alpha}{2}\left(\left.N_{1}\right|_{y=h}+\left.N_{3}\right|_{y=h}\right),
\end{aligned}
$$

where $0<\alpha<1$ is the accommodation coefficient and describes the "roughness" of the wall. $\alpha \rightarrow 0$ implies that the wall is smooth, and therefore particles undergo specular reflection at the walls. In our model this means that incident particles simply bounce off in a direction $90^{\circ}$ from the incident direction. $\alpha \rightarrow 1$ implies that the wall is rough, and therefore particles undergo diffuse reflection at the walls. In our model this means that incident particles are redistributed equally into the two directions pointing away from the wall.

The goal of this section is to derive a $1 \mathrm{D}$ approximation the collisionless equations (2.1) - (2.4) in the case where the channel depth, $h$, is a small parameter. In Sections 2.1-2.3 we derive such a model, in Section 2.4 we indicate how this model behaves in the long-time, and in Section 2.5 we test this 1D model against the full 2D equations in a numerical example. 
2.1. The depth-averaged equations. We begin by defining the depthaveraged number density:

$$
\bar{N}_{i}(x, t)=\frac{1}{h} \int_{0}^{h} N_{i}(x, y, t) d y,
$$

where $i=1,2,3,4$. Depth-averaging equations (2.1)-(2.4) across the channel yields the following equations:

$$
\begin{aligned}
& \frac{\partial \bar{N}_{1}}{\partial t}+\frac{\partial \bar{N}_{1}}{\partial x}=\frac{1}{h}\left(\left.N_{1}\right|_{y=0}-\left.N_{1}\right|_{y=h}\right) \\
& \frac{\partial \bar{N}_{2}}{\partial t}-\frac{\partial \bar{N}_{2}}{\partial x}=\frac{1}{h}\left(\left.N_{2}\right|_{y=h}-\left.N_{2}\right|_{y=0}\right) \\
& \frac{\partial \bar{N}_{3}}{\partial t}-\frac{\partial \bar{N}_{3}}{\partial x}=\frac{1}{h}\left(\left.N_{3}\right|_{y=0}-\left.N_{3}\right|_{y=h}\right) \\
& \frac{\partial \bar{N}_{4}}{\partial t}+\frac{\partial \bar{N}_{4}}{\partial x}=\frac{1}{h}\left(\left.N_{4}\right|_{y=h}-\left.N_{4}\right|_{y=0}\right) .
\end{aligned}
$$

Applying boundary conditions (2.5) - (2.8) yields

$$
\begin{aligned}
& \frac{\partial \bar{N}_{1}}{\partial t}+\frac{\partial \bar{N}_{1}}{\partial x}=\frac{1}{h}\left(\left.(1-\alpha) N_{4}\right|_{y=0}+\frac{\alpha}{2}\left(\left.N_{2}\right|_{y=0}+\left.N_{4}\right|_{y=0}\right)-\left.N_{1}\right|_{y=h}\right) \\
& \frac{\partial \bar{N}_{2}}{\partial t}-\frac{\partial \bar{N}_{2}}{\partial x}=\frac{1}{h}\left(\left.(1-\alpha) N_{3}\right|_{y=h}+\frac{\alpha}{2}\left(\left.N_{1}\right|_{y=h}+\left.N_{3}\right|_{y=h}\right)-\left.N_{2}\right|_{y=0}\right) \\
& \frac{\partial \bar{N}_{3}}{\partial t}-\frac{\partial \bar{N}_{3}}{\partial x}=\frac{1}{h}\left(\left.(1-\alpha) N_{2}\right|_{y=0}+\frac{\alpha}{2}\left(\left.N_{2}\right|_{y=0}+\left.N_{4}\right|_{y=0}\right)-\left.N_{3}\right|_{y=h}\right) \\
& \frac{\partial \bar{N}_{4}}{\partial t}+\frac{\partial \bar{N}_{4}}{\partial x}=\frac{1}{h}\left(\left.(1-\alpha) N_{1}\right|_{y=h}+\frac{\alpha}{2}\left(\left.N_{1}\right|_{y=h}+\left.N_{3}\right|_{y=h}\right)-\left.N_{4}\right|_{y=0}\right) .
\end{aligned}
$$

We define the total density, $\rho$, and the momentum, $m$, as follows:

$$
\begin{aligned}
\rho(x, t) & =\bar{N}_{1}(x, t)+\bar{N}_{2}(x, t)+\bar{N}_{3}(x, t)+\bar{N}_{4}(x, t) \\
m(x, t) & =\bar{N}_{1}(x, t)-\bar{N}_{2}(x, t)-\bar{N}_{3}(x, t)+\bar{N}_{4}(x, t) .
\end{aligned}
$$

Re-writing equations (2.14)-(2.17) in terms of the total density and the momentum yields

$$
\begin{aligned}
& \frac{\partial \rho}{\partial t}+\frac{\partial m}{\partial x}=0 \\
& \frac{\partial m}{\partial t}+\frac{\partial \rho}{\partial x}=\frac{\alpha}{h}\left(\left.N_{2}\right|_{y=0}+\left.N_{3}\right|_{y=h}-\left.N_{4}\right|_{y=0}-\left.N_{1}\right|_{y=h}\right) .
\end{aligned}
$$

2.2. The thin-channel approximation. We now turn our attention to replacing the right-hand side of equation (2.21) by something only involving $\rho(x, t)$ and $m(x, t)$. We begin by defining the following functions:

$$
\begin{aligned}
& f_{i}(x, y, t)=\frac{1}{h} \int_{0}^{y} N_{i}(x, \eta, t) d \eta \\
& g_{i}(x, y, t)=-\frac{1}{h} \int_{h}^{y} N_{i}(x, \eta, t) d \eta,
\end{aligned}
$$


for $i=1,2,3,4$. Note that

$$
f_{i}(x, h, t)=g_{i}(x, 0, t)=\bar{N}_{i}(x, t) .
$$

In order to relate $f_{i}(x, 0, t)$ to $f_{i}(x, h, t)$, a Taylor expansion about the point $y=0$ can be applied:

$$
\begin{aligned}
f_{i}(x, h, t)=\bar{N}_{i}(x, t) & =f_{i}(x, 0, t)+h \frac{\partial f_{i}}{\partial y}(x, 0, t)+\frac{h^{2}}{2} \frac{\partial^{2} f_{i}}{\partial y^{2}}(x, 0, t)+\ldots \\
& =0+N_{i}(x, 0, t)+\frac{h}{2} \frac{\partial N_{i}}{\partial y}(x, 0, t)+\ldots
\end{aligned}
$$

Similarly, in order to relate $g_{i}(x, h, t)$ to $g_{i}(x, 0, t)$, a Taylor expansion about the point $y=h$ can be applied:

$$
\begin{aligned}
g_{i}(x, 0, t)=\bar{N}_{i}(x, t) & =g_{i}(x, h, t)-h \frac{\partial g_{i}}{\partial y}(x, h, t)+\frac{h^{2}}{2} \frac{\partial^{2} g_{i}}{\partial y^{2}}(x, h, t)+\ldots \\
& =0+N_{i}(x, h, t)-\frac{h}{2} \frac{\partial N_{i}}{\partial y}(x, h, t)+\ldots
\end{aligned}
$$

Next, we can replace the partial derivatives with respect to $y$ in $(2.24)-(2.25)$ by appealing to the two-dimensional equations (2.1)-(2.4); noting that

$$
N_{i}=\bar{N}_{i}+\mathcal{O}(h)
$$

we find:

$$
\begin{aligned}
-\frac{\partial N_{1}}{\partial y}(x, h, t) & =\left(\frac{\partial \bar{N}_{1}}{\partial t}+\frac{\partial \bar{N}_{1}}{\partial x}\right)+\mathcal{O}(h) \\
-\frac{\partial N_{3}}{\partial y}(x, h, t) & =\left(\frac{\partial \bar{N}_{3}}{\partial t}+\frac{\partial \bar{N}_{3}}{\partial x}\right)+\mathcal{O}(h) \\
\frac{\partial N_{2}}{\partial y}(x, 0, t) & =\left(\frac{\partial \bar{N}_{2}}{\partial t}+\frac{\partial \bar{N}_{2}}{\partial x}\right)+\mathcal{O}(h) \\
\frac{\partial N_{4}}{\partial y}(x, 0, t) & =\left(\frac{\partial \bar{N}_{4}}{\partial t}+\frac{\partial \bar{N}_{4}}{\partial x}\right)+\mathcal{O}(h) .
\end{aligned}
$$

Combining the above result and the Taylor expansions (2.24) and (2.25) yields the following result:

$$
\begin{aligned}
& \left.N_{1}\right|_{y=h}=\bar{N}_{1}-\frac{h}{2}\left(\frac{\partial \bar{N}_{1}}{\partial t}+\frac{\partial \bar{N}_{1}}{\partial x}\right)+\mathcal{O}\left(h^{2}\right) \\
& \left.N_{3}\right|_{y=h}=\bar{N}_{3}-\frac{h}{2}\left(\frac{\partial \bar{N}_{3}}{\partial t}-\frac{\partial \bar{N}_{3}}{\partial x}\right)+\mathcal{O}\left(h^{2}\right) \\
& \left.N_{2}\right|_{y=0}=\bar{N}_{2}-\frac{h}{2}\left(\frac{\partial \bar{N}_{2}}{\partial t}-\frac{\partial \bar{N}_{2}}{\partial x}\right)+\mathcal{O}\left(h^{2}\right) \\
& \left.N_{4}\right|_{y=0}=\bar{N}_{4}-\frac{h}{2}\left(\frac{\partial \bar{N}_{4}}{\partial t}+\frac{\partial \bar{N}_{4}}{\partial x}\right)+\mathcal{O}\left(h^{2}\right) .
\end{aligned}
$$

Finally, this result can be plugged into (2.21) to produce

$$
\frac{\partial m}{\partial t}+\frac{\partial \rho}{\partial x}=\frac{\alpha}{2}\left(\frac{\partial m}{\partial t}+\frac{\partial \rho}{\partial x}\right)-\frac{\alpha}{h} m+\mathcal{O}(h) .
$$


Therefore, to order $\mathcal{O}(h)$ we obtain the following system of equations:

$$
\begin{aligned}
& \frac{\partial \rho}{\partial t}+\frac{\partial m}{\partial x}=0 \\
& \frac{\partial m}{\partial t}+\frac{\partial \rho}{\partial x}=\left(\frac{-2 \alpha}{2-\alpha}\right)\left(\frac{1}{h}\right) m .
\end{aligned}
$$

We will refer to this system of hyperbolic partial differential equations as the collisionless thin-channel model.

2.3. A telegraph equation. Further insight can be gained into the collisionless thin-channel model by taking the derivative of equation (2.27) with respect to $t$ and the derivative of equation (2.28) with respect to $x$ :

$$
\begin{aligned}
& \frac{\partial^{2} \rho}{\partial t^{2}}+\frac{\partial^{2} m}{\partial x \partial t}=0 \\
& \frac{\partial^{2} m}{\partial x \partial t}+\frac{\partial^{2} \rho}{\partial x^{2}}=\left(\frac{-2 \alpha}{2-\alpha}\right)\left(\frac{1}{h}\right) \frac{\partial m}{\partial x} .
\end{aligned}
$$

Combining these two equations and reordering the terms produces the following telegraph equation:

$$
\left(\frac{2 \alpha}{2-\alpha}\right) \frac{\partial \rho}{\partial t}-h \frac{\partial^{2} \rho}{\partial x^{2}}+h \frac{\partial^{2} \rho}{\partial t^{2}}=0 .
$$

Therefore, we have shown that the collisionless thin-channel model is in fact a telegraph equation. This implies that the depth-averaged density, $\rho(x, t)$, has the tendency to both propagate and diffuse along the channel. The derivation we have presented in this section is not rigorous; however, in Section 4 we will in fact prove that the 2D 4 -velocity Broadwell model converges weakly to the telegraph equation (2.31) under an appropriate scaling (Theorem 4.2).

2.4. Long-time behavior: the diffusion equation. It is well-known that as $t \rightarrow \infty$, the telegraph equation on an infinite domain behaves like a diffusion equation (see for example Zauderer [35]). In Section 4 we will prove that under an appropriate scaling, the 2D 4-velocity Broadwell model converges weakly to a diffusion equation. In this section, however, we will use a non-rigorous approach to obtain a diffusion equation from the telegraph equation (2.31).

We consider the behavior of system $(2.27)-(2.28)$ on a time scale of $t=\mathcal{O}\left(h^{-1}\right)$. On this time scale, equation (2.28) reduces to the following relationship:

$$
\mathcal{O}(h)+\frac{\partial \rho}{\partial x}=\left(\frac{-2 \alpha}{2-\alpha}\right)\left(\frac{1}{h}\right) m .
$$

Solving this equation for $m(x, t)$ yields

$$
m=-h\left(\frac{2-\alpha}{2 \alpha}\right) \frac{\partial \rho}{\partial x}+\mathcal{O}\left(h^{2}\right) .
$$

Substituting this result into the conservation of mass equation, $(2.27)$, yields to $\mathcal{O}\left(h^{2}\right)$ the following diffusion equation:

$$
\frac{\partial \rho}{\partial t}-h\left(\frac{2-\alpha}{2 \alpha}\right) \frac{\partial^{2} \rho}{\partial x^{2}}=0 .
$$


Therefore, on long-time scales the collisionless thin-channel model approximately diffuses down the channel with the diffusion constant proportional to the channel depth, $h$. This result is qualitatively in agreement with previous work on the long-time behavior of a Knudsen gas in a thin channel [4, 5, 21].

2.5. An example. In order to demonstrate the validity of the above derived collisionless thin-channel model, we consider an example with $h=0.04, \alpha=0.5$, and the following initial conditions:

$$
N_{1,2,3,4}(x, y, 0)= \begin{cases}0.25 & \text { if }(x-0.5)^{2}+15(y-0.02)^{2}<0.15^{2} \\ 0 & \text { otherwise }\end{cases}
$$

The solution to the $2 \mathrm{D}$ collisionless 4 -velocity Broadwell model is computed on a $1000 \times 20$ grid on the computational domain $[-0.5,1.5] \times[0, h]$ using the CLAWPACK software package [23], which is based on the wave propagation method of LeVeque [24]. We take in this computation a CFL (Courant-Friedrichs-Lewy) number of exactly one:

$$
\frac{\Delta t}{\Delta x}=\frac{\Delta t}{\Delta y}=1,
$$

which for this constant coefficient problem results in the wave propagation method reproducing the exact solution. Ghost cells are introduced to enforce boundary conditions (2.5)-(2.8) at $y=0$ and $y=h$ and zeroth-order extrapolation conditions at $x=-0.5$ and $x=1.5[25]$.

The thin-channel model (2.27)-(2.28) with initial conditions

$$
\begin{aligned}
\rho(x, 0) & = \begin{cases}\frac{10}{3} \sqrt{0.15^{2}-(x-0.5)^{2}} & \text { if } 0.35<x<0.65 \\
0 & \text { otherwise }\end{cases} \\
m(x, 0) & =0
\end{aligned}
$$

is also solved using the CLAWPACK software package. In particular we use a grid of 2000 points on the computational domain $[-0.5,1.5]$, zeroth-order extrapolation conditions at $x=-0.5$ and $x=1.5$, and a CFL number of 0.95 . The source term in (2.28) is coupled to the left-hand side of system (2.27)-(2.28) through Strang operator splitting [25]. The results of this $1 \mathrm{D}$ simulation are compared against the depthaveraged 2D solution as well as the exact solution of the diffusion equation (2.34) in Figures $3.1-3.2$. In these figures we only display the solution for $x \in[0.3,0.7]$. These results show that the 1D thin-channel model accurately captures the general form of the depth-averaged 2D solution. The plots also indicate that the diffusion equation is not accurate for short times, but becomes more accurate for long time. Finally, these plots show that for a fixed $h$ and $t \rightarrow \infty$ the 1D thin-channel model converges only weakly the Broadwell model solution. In Section 4 we will rigorously prove that the convergence is indeed only weak.

\section{Derivation of a collisional thin-channel model}

We now attempt to obtain an analogous result to the above thin-channel model in the case of the collisional Broadwell model. The Broadwell model with collisions is 
given by

$$
\begin{aligned}
\frac{\partial N_{1}}{\partial t}+\frac{\partial N_{1}}{\partial x}+\frac{\partial N_{1}}{\partial y} & =\frac{1}{3} \sigma\left(-2 N_{1} N_{2}+N_{3} N_{4}+N_{5}^{2}\right) \\
\frac{\partial N_{2}}{\partial t}-\frac{\partial N_{2}}{\partial x}-\frac{\partial N_{2}}{\partial y} & =\frac{1}{3} \sigma\left(-2 N_{1} N_{2}+N_{3} N_{4}+N_{5}^{2}\right) \\
\frac{\partial N_{3}}{\partial t}-\frac{\partial N_{3}}{\partial x}+\frac{\partial N_{3}}{\partial y} & =\frac{1}{3} \sigma\left(N_{1} N_{2}-2 N_{3} N_{4}+N_{5}^{2}\right) \\
\frac{\partial N_{4}}{\partial t}+\frac{\partial N_{4}}{\partial x}-\frac{\partial N_{4}}{\partial y} & =\frac{1}{3} \sigma\left(N_{1} N_{2}-2 N_{3} N_{4}+N_{5}^{2}\right) \\
\frac{\partial N_{5}}{\partial t} & =\frac{1}{3} \sigma\left(N_{1} N_{2}+N_{3} N_{4}-2 N_{5}^{2}\right),
\end{aligned}
$$

where $\sigma \geq 0$ is the collision constant and is related to the dimensional collision constant in the following way:

$$
\sigma^{\star}=L^{2} c \sigma
$$

$N_{5}$ represents the velocities in both the + and $-z$-directions. The collisions that appear on the right-hand sides of these equations can be understood by noting the following properties:

1. The only collisions that actually change the densities are $N_{1}-N_{2}, N_{3}-N_{4}$, and $N_{5}-N_{5}$

2. Each of these collisions will produce one the following three outcomes each with a $1 / 3$ probability: $N_{1}$ and $N_{2}, N_{3}$ and $N_{4}$, and $N_{5}$ and $N_{5}$.

Therefore, the right-hand side of (3.1), for example, says that $N_{1}$ will be produced with probability $1 / 3$ if an $N_{3}-N_{4}$ collision occurs, produced with probability $1 / 3$ if an $N_{5}-N_{5}$ collision occurs, but lost with probability $2 / 3$ if an $N_{1}-N_{2}$ collision occurs. The remaining right-hand sides follow from similar reasoning for $N_{2}$ through $N_{5}$. We should note here that equation (3.5) represents only one of the $N_{5}$ velocities (i.e., only the + or only the $-z$-direction density); and therefore, to obtain the total density of all particles we will have to count $N_{5}$ twice.

The goal of this section is to derive a 1D approximation the collisional equations (3.1) - (3.5) in the case where the channel depth, $h$, is a small parameter. In Section 3.1 we derive such a model, in Section 3.2 we indicate how this model behaves in the long-time, and in Section 3.3 we test this 1D model against the full 2D equations in a numerical example.

3.1. The thin-channel model. We begin, again, by depth-averaging the above equations. The key observation one needs to make is that

$$
\sigma \overline{N_{i} N_{j}} \equiv \frac{\sigma}{h} \int_{0}^{h} N_{i}(x, y, t) N_{j}(x, y, t) d y=\sigma \bar{N}_{i} \bar{N}_{j}+\mathcal{O}\left(\sigma h^{2}\right) .
$$

With this approximation, it is quite straightforward to use the same techniques as in the collisionless case to obtain the thin-channel approximation. However, in order to obtain a thin-channel approximation that is $\mathcal{O}(h)$, we make the following assumption:

$$
\sigma \leq \mathcal{O}\left(h^{-1}\right),
$$

which gives an upper bound on the collision constant in order to still obtain quasi-1D solutions. Under this assumption, the approximation in (3.6) is always accurate to at 
least $\mathcal{O}(h)$. The total density, $\rho$, and the momentum, $m$, can be defined as

$$
\begin{aligned}
\rho(x, t) & =\bar{N}_{1}(x, t)+\bar{N}_{2}(x, t)+\bar{N}_{3}(x, t)+\bar{N}_{4}(x, t)+2 \bar{N}_{5}(x, t) \\
m(x, t) & =\bar{N}_{1}(x, t)-\bar{N}_{2}(x, t)-\bar{N}_{3}(x, t)+\bar{N}_{4}(x, t) .
\end{aligned}
$$

By doing the identical steps as before, the Broadwell equations with collisions can be reduced to the following system of first order partial differential equations:

$$
\begin{aligned}
\frac{\partial \rho}{\partial t}+\frac{\partial m}{\partial x} & =0 \\
\frac{\partial m}{\partial t}+\frac{\partial}{\partial x}(\rho-2 z) & =\left(\frac{-2 \alpha}{2-\alpha}\right)\left(\frac{1}{h}\right) m \\
\frac{\partial z}{\partial t} & =\frac{\sigma}{24}\left((\rho+2 z)(\rho-6 z)-m^{2}\right),
\end{aligned}
$$

where $z(x, t) \equiv \bar{N}_{5}(x, t)$. We will refer to this system of hyperbolic partial differential equations as the collisional thin-channel model. The right-hand side of equation (3.12) was obtained by noting that if the depth-averaged $y$-momentum is zero then $\bar{N}_{1}=\bar{N}_{4}$ and $\bar{N}_{2}=\bar{N}_{3}$. System (3.10)-(3.12) is analogous to the collisionless system (2.27)-(2.28); however, the collisional system cannot be written as a simple telegraph equation analogous to $(2.31)$.

3.2. Long-time behavior: the diffusion equation. Let us consider the behavior of system (3.10)-(3.12) on a time scale of $t=\mathcal{O}\left(h^{-p}\right)$, where $p \geq 1$. We note that the value of $p$ should be made large enough in order to allow equation (3.12) to relax. Therefore, $p$ directly depends on the strength of $\sigma$ : the weaker the collisions, the larger $p$ has to be in order for the equation to (3.12) to relax to its natural state:

$$
\frac{h^{p}}{\sigma} \ll 1
$$

On this time scale, equations (3.11) and (3.12) reduce to the following relationships:

$$
\begin{aligned}
\mathcal{O}\left(h^{p}\right)+\frac{\partial}{\partial x}(\rho-2 z) & =\left(\frac{-2 \alpha}{2-\alpha}\right)\left(\frac{1}{h}\right) m \\
\mathcal{O}\left(\frac{h^{p}}{\sigma}\right) & =(\rho+2 z)(\rho-6 z)-m^{2} .
\end{aligned}
$$

Solving these equations to leading for $z(x, t)$ and $m(x, t)$ yields

$$
\begin{aligned}
z & =\frac{\rho}{6}+\cdots \\
m & =-\frac{2}{3} h\left(\frac{2-\alpha}{2 \alpha}\right) \frac{\partial \rho}{\partial x}+\cdots .
\end{aligned}
$$

Substituting this result into the conservation of mass equation, (3.10), yields the following diffusion equation:

$$
\frac{\partial \rho}{\partial t}-\frac{2}{3} h\left(\frac{2-\alpha}{2 \alpha}\right) \frac{\partial^{2} \rho}{\partial x^{2}}=0 .
$$

Note that the diffusion constant in (3.17) is 1.5 times smaller than the diffusion constant in (2.34) and is independent of the actual value of $\sigma$ (as long as $\sigma<\mathcal{O}\left(h^{-1}\right)$ ). 
This sudden jump to $2 / 3$ of the collisionless value can be explained by the fact that the Broadwell model goes from being described entirely by four directions in the collisionless case to six when collisions are included. These extra directions allow for some mass to accumulate in $N_{5}=N_{6}$, and unlike the other directions, $N_{5}$ and $N_{6}$ do not contribute to the quantity that is being damped by wall collisions: the $x$-momentum.

Unlike in the collisionless case, we have no rigorous theorems to show that the collisional Broadwell model converges either to system (3.10)-(3.12) or to diffusion equation (3.17). However, we will provide numerical evidence below that suggests that system (3.10)-(3.12) is a good approximation to the collisional Broadwell model when $h$ is small; and furthermore, that diffusion equation (3.17) is a good approximation to both (3.10)-(3.12) and the Broadwell model in the long-time.

3.3. An example. In order to demonstrate the validity of the above derived collisional thin-channel model, we consider an example with $h=0.04, \alpha=0.5, \sigma=100$, and the following initial conditions:

$$
\begin{aligned}
N_{1,2,3,4}(x, y, 0) & = \begin{cases}0.25 & \text { if }(x-0.5)^{2}+15(y-0.02)^{2}<0.15^{2} \\
0 & \text { otherwise }\end{cases} \\
N_{5}(x, y, 0) & =0
\end{aligned}
$$

The solution to the 2D collisional 4-velocity Broadwell model is computed on the same computational grid with the same boundary conditions as in Section 2.5. We take in this computation a CFL number of 0.995 :

$$
\frac{\Delta t}{\Delta x}=\frac{\Delta t}{\Delta y}=0.995 .
$$

The collisional source term is approximated via the implicit trapezoidal rule and coupled to the left-hand side of system (3.1)-(3.5) through Strang operator splitting.

The thin-channel model (3.10)-(3.12) with initial conditions

$$
\begin{aligned}
\rho(x, 0) & = \begin{cases}\frac{10}{3} \sqrt{0.15^{2}-(x-0.5)^{2}} & \text { if } 0.35<x<0.65 \\
0 & \text { otherwise }\end{cases} \\
m(x, 0) & =0 \\
z(x, 0) & =0
\end{aligned}
$$

is solved on a grid of 2000 points on the computational domain [-0.5, 1.5$]$. The source term in (2.28) is again approximated using the implicit trapezoidal rule and coupled to the left-hand side of system (3.10)-(3.12) through Strang operator splitting. The results of this $1 \mathrm{D}$ simulation are compared against the depth-averaged $2 \mathrm{D}$ solution as well as the exact solution of the diffusion equation (3.17) in Figures 3.3-3.4. In these figures we only display the solution for $x \in[0,1]$. These results show that the $1 \mathrm{D}$ thinchannel model accurately captures the depth-averaged behavior of the 2D solution; and furthermore, they indicate that the diffusion equation is a good approximation only in the long-time. 
(a)

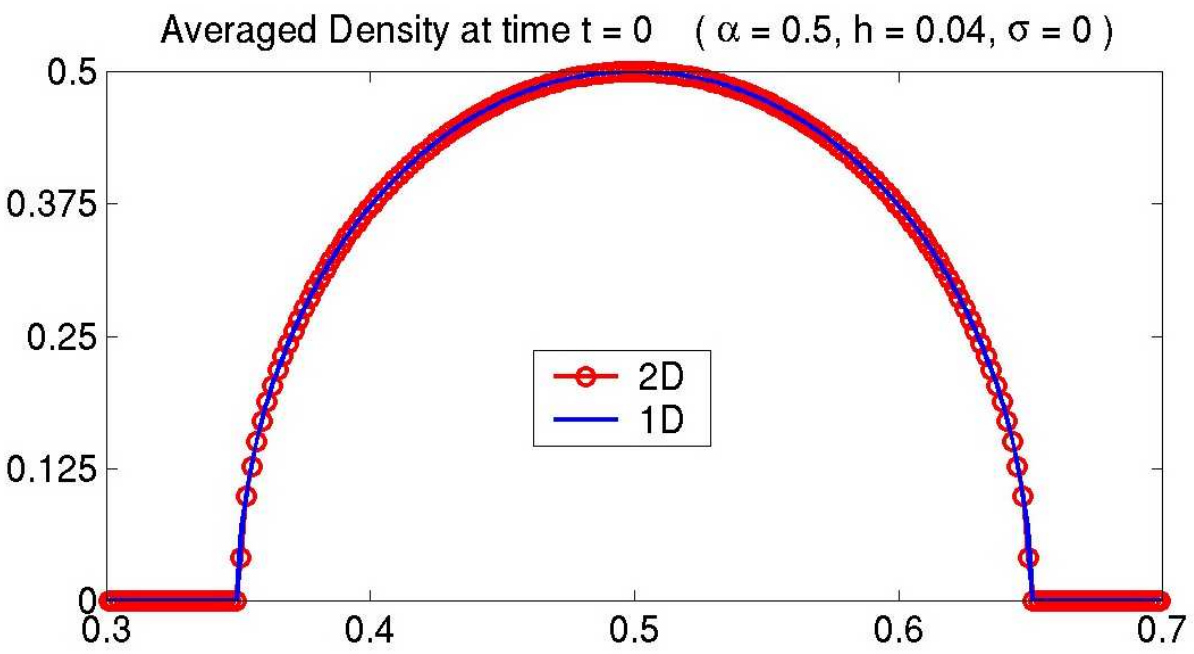

Averaged Density at time $t=0.2 \quad(\alpha=0.5, h=0.04, \sigma=0)$

(b)

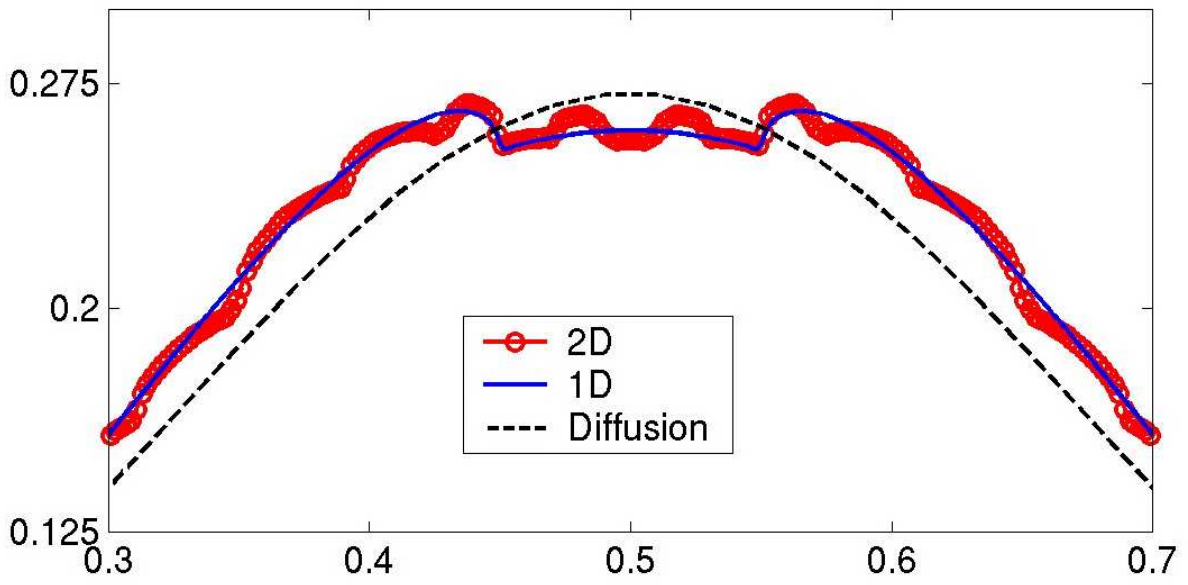

Averaged Density at time $t=0.4 \quad(\alpha=0.5, h=0.04, \sigma=0)$

(c)

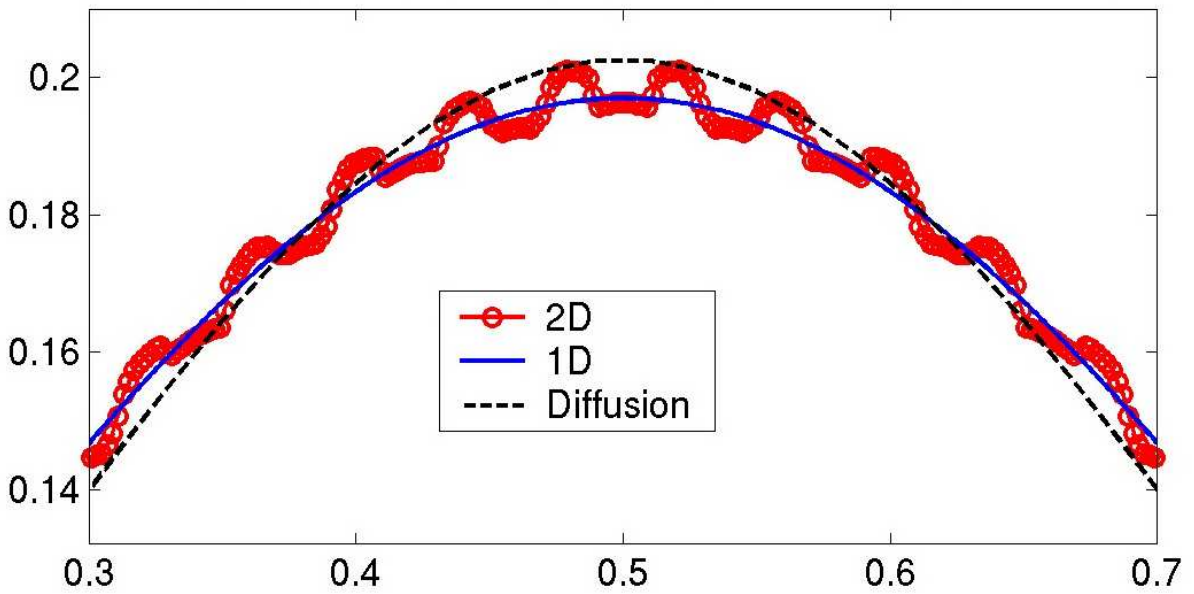

FIG. 3.1. The Broadwell model without collisions $(\sigma=0)$ in a thin channel. Shown in these panels are the depth-averaged exact $2 D$ solution (circles-line), $1 D$ telegraph solution (solid line), $1 D$ diffusion solution (dashed line). The exact $2 D$ solution is sampled on a $1000 \times 20$ grid for $(x, y) \in$ $[-0.5,1.5] \times[0,0.04]$, the approximate $1 D$ solution is computed with 2000 points for $x \in[-0.5,1.5]$, and the diffusion equation is solved exactly. 
(a)

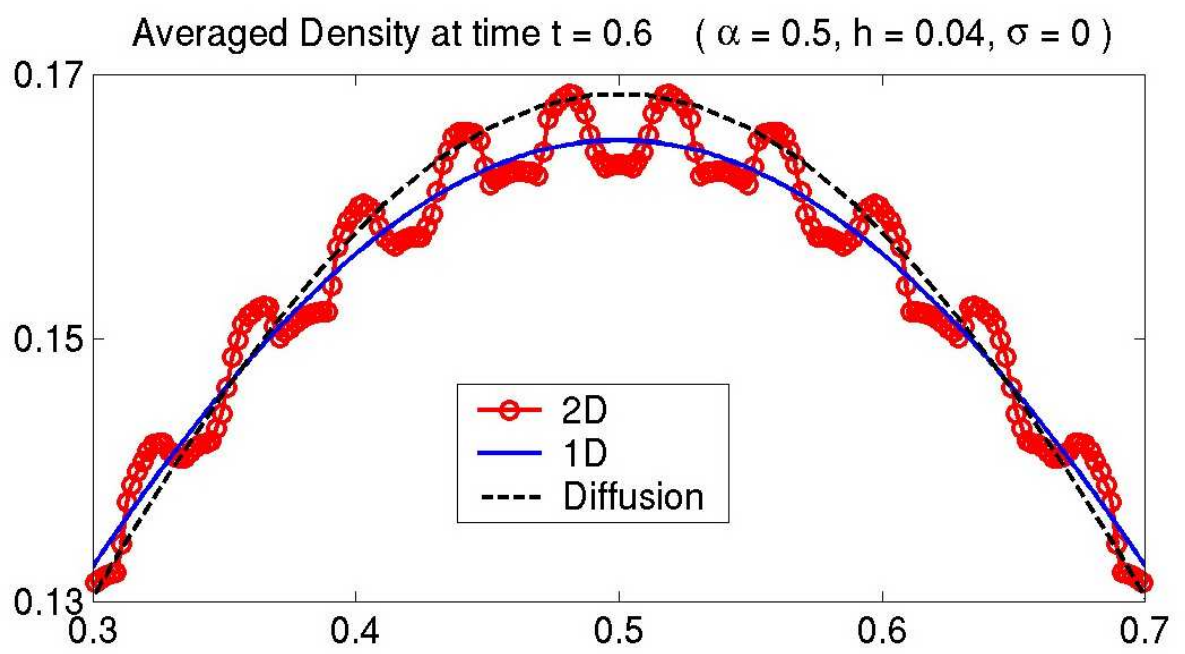

Averaged Density at time $\mathrm{t}=0.8 \quad(\alpha=0.5, \mathrm{~h}=0.04, \sigma=0)$

(b)

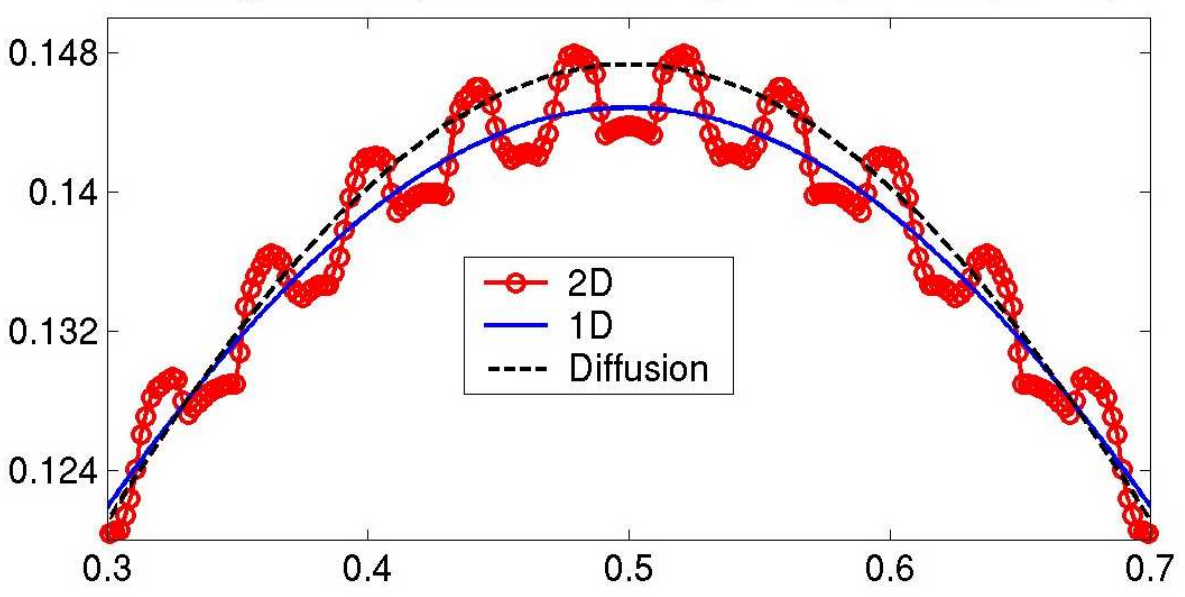

Averaged Density at time $t=1 \quad(\alpha=0.5, h=0.04, \sigma=0)$

(c)

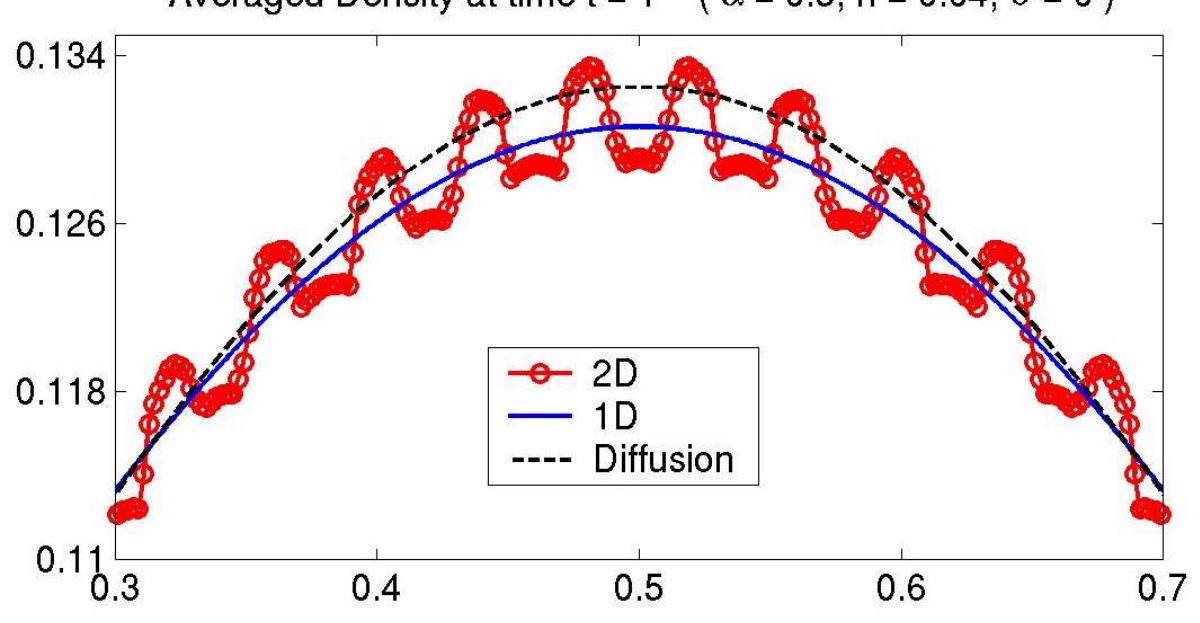

FIG. 3.2. Collisionless Broadwell solution continued from Figure 3.1. One can see from these plots that the convergence of the $1 D$ thin-channel approximation to the depth-averaged $2 D$ system for a fixed $h$ and $t \rightarrow \infty$ is only weak. 
(a)

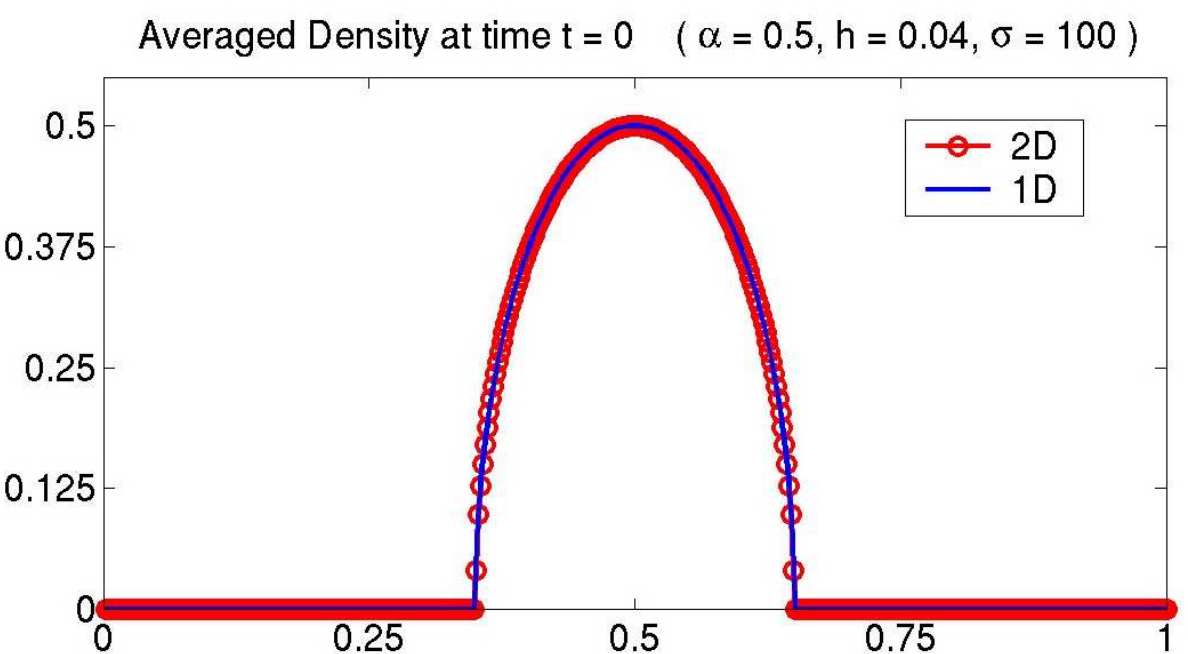

Averaged Density at time $t=0.2 \quad(\alpha=0.5, \mathrm{~h}=0.04, \sigma=100)$

(b)

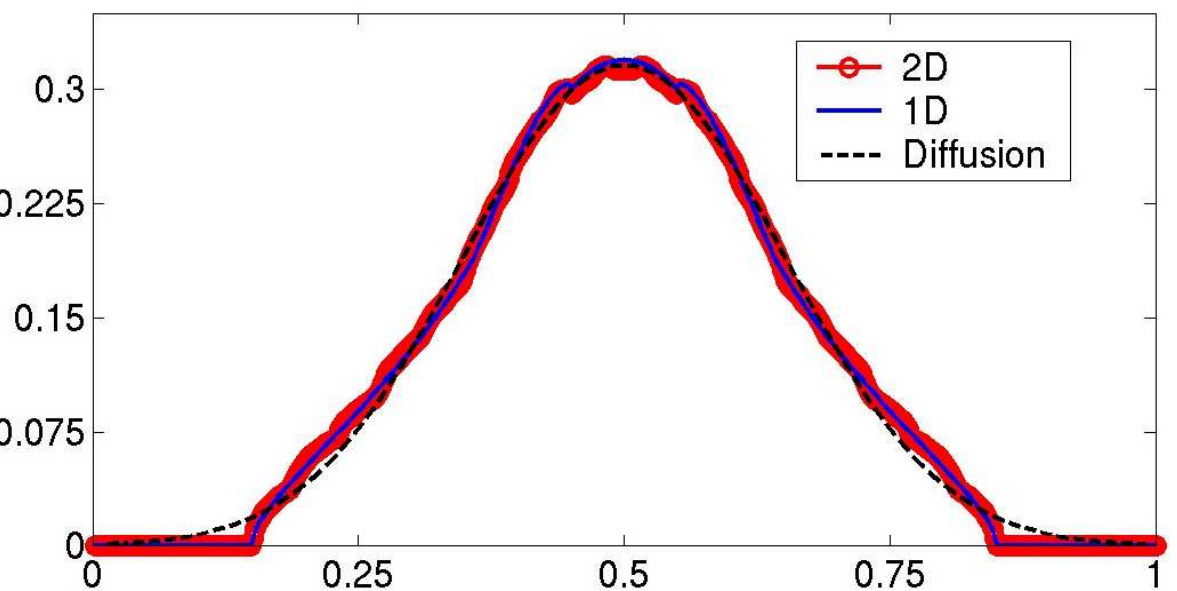

Averaged Density at time $\mathrm{t}=0.4 \quad(\alpha=0.5, \mathrm{~h}=0.04, \sigma=100)$

(c)

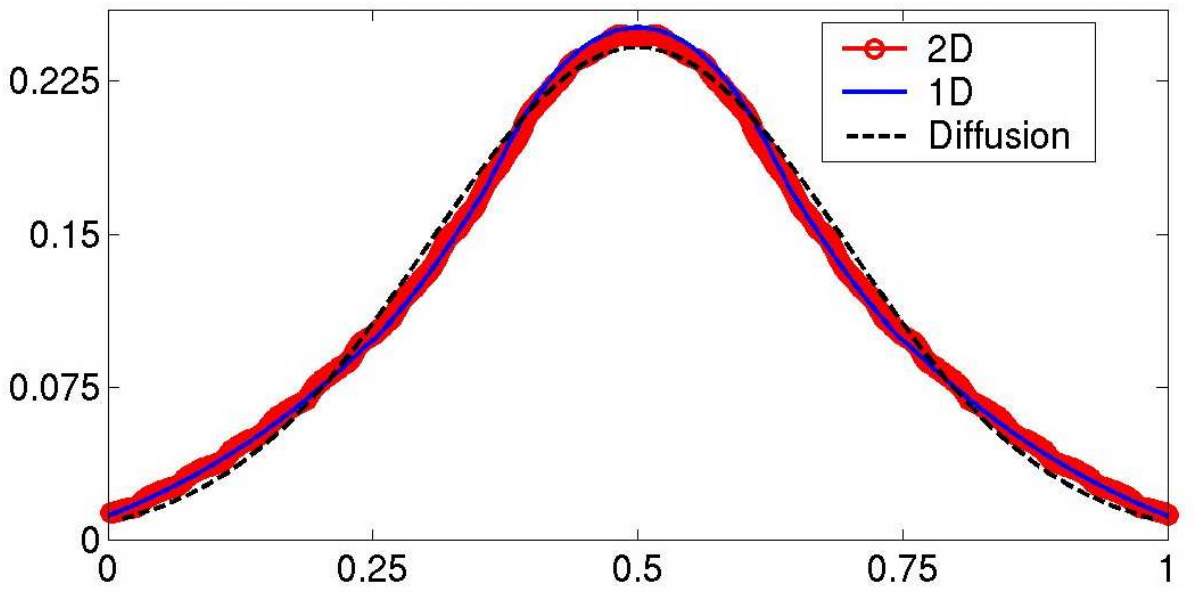

FIG. 3.3. The Broadwell model with collisions $(\sigma=100)$ in a thin channel. Shown in these panels are the depth-averaged exact $2 D$ solution (circles-line), 1D telegraph solution (solid line), 1D diffusion solution (dashed line). The exact $2 D$ solution is sampled on a $1000 \times 20$ grid for $(x, y) \in$ $[-0.5,1.5] \times[0,0.04]$, the approximate $1 D$ solution is computed with 2000 points for $x \in[-0.5,1.5]$, and the diffusion equation is solved exactly. 
(a)
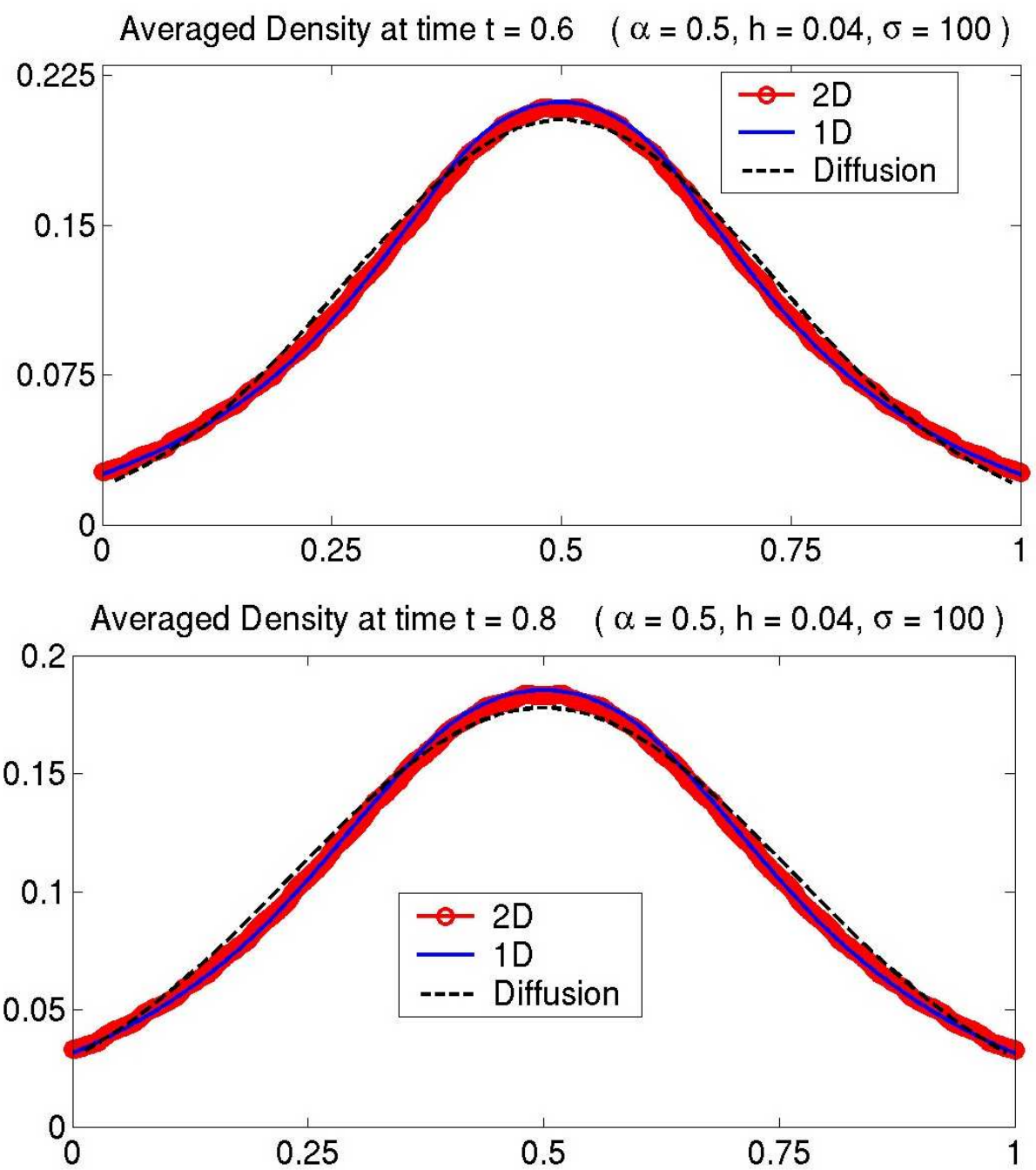

(b)

Averaged Density at time $t=1 \quad(\alpha=0.5, h=0.04, \sigma=100)$

(c)

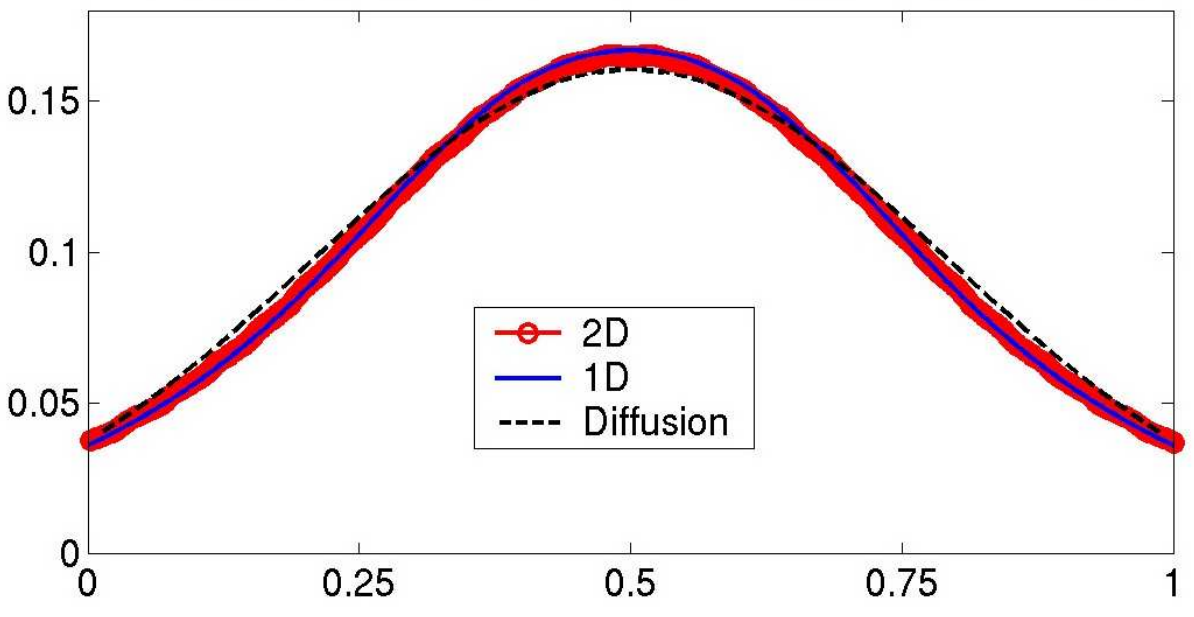

FIG. 3.4. Collisional Broadwell solution continued from Figure 3.3. 


\section{Limiting behavior theorems in the collisionless case}

In this section we shall prove some theorems concerning limiting behavior of the 4 -velocity collisionless Broadwell model (2.1)-(2.4). To simplify the presentation we shall rescale $x, y$, and $t$ so that $h=1$ and the domain is $\mathrm{D}=\{(x, y) \mid(-\infty, \infty) \times[0,1]\}$ in this section only. We shall also write the unknowns as a vector,

$$
\boldsymbol{N}(x, y, t)=\left(N_{1}, N_{2}, N_{3}, N_{4}\right)^{T} .
$$

It is useful to define the inner product,

$$
\langle\boldsymbol{u}, \boldsymbol{w}\rangle \equiv \int_{0}^{1} \boldsymbol{u}^{H} \boldsymbol{w} d y,
$$

and the vectors $\mathbf{1} \equiv(1,1,1,1)^{T}$ and $\mathbf{1}_{ \pm} \equiv(1,-1,-1,1)^{T}$. The depth-averaged density and momentum can now be written as follows:

$$
\begin{aligned}
\rho(x, t) & =\langle\mathbf{1}, \boldsymbol{N}(x, \cdot, t)\rangle \\
m(x, t) & =\left\langle\mathbf{1}_{ \pm}, \boldsymbol{N}(x, \cdot, t)\right\rangle .
\end{aligned}
$$

We will also require the Fourier transform of $\boldsymbol{N}$ in the $x$-direction, which we denote as $\widehat{N}$ and define as follows:

$$
\widehat{\boldsymbol{N}}(k, y, t)=\frac{1}{2 \pi} \int_{-\infty}^{\infty} \boldsymbol{N}(x, y, t) e^{-i k x} d x .
$$

We shall use the following function space for our theorems

$$
\boldsymbol{N} \in \boldsymbol{B}(\mathrm{D}) \quad \text { if } \quad \max _{0 \leq y \leq 1} \int_{-\infty}^{\infty}\left(|\boldsymbol{N}(x, y)|+|\boldsymbol{N}(x, y)|^{2}+\left|\boldsymbol{N}_{x}(x, y)\right|^{2}\right) d x<\infty
$$

In other words, $\boldsymbol{N}(x, y) \in L^{1} \cap H^{1}(\mathrm{R})$ for each $y \in[0,1]$.

4.1. Diffusive behavior. The discussion in Section 2 suggests that the Broadwell model under certain conditions behaves like a diffusion equation. We shall prove that under a diffusive scaling the depth-averaged density converges weakly to a solution of a diffusion equation.

Suppose we consider initial conditions of the form

$$
\boldsymbol{N}(x, y, 0)=\boldsymbol{M}_{0}(x / \lambda, y) / \lambda,
$$

where $\lambda$ is a length scale. We note that the total mass for these initial conditions is independent of $\lambda$. We use the following rescaled independent variables:

$$
X=x / \lambda \text { and } T=t / \lambda^{2} .
$$

In these variables, our new unknown is the rescaled number density, denoted by $\boldsymbol{M}_{\lambda}(X, y, T)$ and it is related to the original density by

$$
\boldsymbol{M}_{\lambda}(X, y, T) \equiv \lambda \boldsymbol{N}\left(\lambda X, y, \lambda^{2} T\right),
$$

where the initial conditions are

$$
\boldsymbol{M}_{\lambda}(X, y, 0)=\boldsymbol{M}_{0}(X, y) .
$$


The rescaled depth-averaged density is denoted $\psi_{\lambda}(X, T)$ and is given by

$$
\psi_{\lambda}(X, T)=\left\langle\mathbf{1}, \boldsymbol{M}_{\lambda}(X, \cdot, T)\right\rangle .
$$

We have the following theorem concerning the behavior of the rescaled density in the limit as $\lambda \rightarrow \infty$.

Theorem 4.1. If the initial conditions are $\boldsymbol{N}(x, y, 0)=\boldsymbol{M}_{0}(x / \lambda, y) / \lambda$, where $\boldsymbol{M}_{0}(x, y) \in \boldsymbol{B}(\mathrm{D})$, then as $\lambda \rightarrow \infty, \psi_{\lambda}(X, T)$ (defined by (4.7)) converges weakly to $\psi(X, T)$ which satisfies the diffusion equation:

$$
\psi_{T}=D \psi_{X X},
$$

where $D=(2-\alpha) / 2 \alpha$, with the initial condition

$$
\psi(X, 0)=\left\langle\mathbf{1}, \boldsymbol{M}_{0}(X, \cdot)\right\rangle .
$$

This theorem establishes that under a diffusive scaling the depth-averaged density for the Broadwell model satisfies (weakly) a diffusion equation.

4.2. Hyperbolic behavior. The results from Section 2 also suggest that the density for the Broadwell model should have a connection with a telegraph equation. Here we verify that under hyperbolic scaling the depth-averaged density converges weakly to a solution of the telegraph equation. To explain this statement more precisely, we first recall the telegraph equation (2.31),

$$
\frac{2 \alpha}{(2-\alpha)} \rho_{t}-\rho_{x x}+\rho_{t t}=0,
$$

where we have set $c=h=1$. Next we use the following rescaling:

$$
X=x / \lambda \text { and } T=t / \lambda,
$$

and obtain the following telegraph equation:

$$
\frac{2 \alpha \lambda}{(2-\alpha)} u_{T}-u_{X X}+u_{T T}=0
$$

where $u(X, T)=\lambda \rho(\lambda X, \lambda T)$. We note that if we let $\alpha \rightarrow 0$ and $\lambda \rightarrow \infty$ so that $\alpha \lambda=2 \Gamma$, we obtain that

$$
2 \Gamma u_{T}-u_{X X}+u_{T T}=0 .
$$

For later reference we note that the solution of this equation can be written as

$$
u(X, T)=\int_{-\infty}^{\infty} \widehat{g}_{1}(k) e^{i\left(k X-\sigma_{1}(k) T\right)} d k+\int_{-\infty}^{\infty} \widehat{g}_{2}(k) e^{i\left(k X-\sigma_{2}(k) T\right)} d k
$$

where

$$
\sigma_{1,2}(k)=-i \Gamma \pm i \sqrt{\Gamma^{2}-k^{2}}
$$

and $\widehat{g}_{1}$ and $\widehat{g}_{2}$ are determined by initial conditions. In particular if $\rho(X, 0)=\rho_{0}(X)$ and $m(X, 0)=m_{0}(X)$, then

$$
\widehat{g}_{1}=\frac{\sigma_{2} \widehat{\rho}_{0}-k \widehat{m}_{0}}{\sigma_{2}-\sigma_{1}} \quad \text { and } \quad \widehat{g}_{2}=\frac{\sigma_{1} \widehat{\rho}_{0}-k \widehat{m}_{0}}{\sigma_{1}-\sigma_{2}} .
$$


Based on the scalings used above we define the following rescaled number density:

$$
\boldsymbol{P}_{\lambda}(X, y, T) \equiv \lambda \boldsymbol{N}(\lambda X, y, \lambda T, \alpha=2 \Gamma / \lambda),
$$

and the rescaled depth-averaged density:

$$
\phi_{\lambda}(X, T)=\left\langle\mathbf{1}, \boldsymbol{P}_{\lambda}(X, \cdot, T)\right\rangle .
$$

We can prove the following theorem concerning the telegraph equation and the Broadwell model.

Theorem 4.2. If the initial conditions are $\boldsymbol{N}(x, y, 0)=\boldsymbol{M}_{0}(x / \lambda, y) / \lambda$, where $\boldsymbol{M}_{0}(x, y) \in \boldsymbol{B}(\mathrm{D})$, then as $\lambda \rightarrow \infty$ and $\alpha \rightarrow 0$ with $\alpha \lambda=2 \Gamma$ we have that $\phi_{\lambda}(X, T)$ converges weakly to $\phi(X, T) . \phi(X, T)$ is a solution of the telegraph equation given by (4.9) with initial conditions:

$$
\begin{aligned}
\phi(X, 0) & =\left\langle\mathbf{1}, \boldsymbol{M}_{0}(X, \cdot)\right\rangle \quad \text { and } \\
m(X, 0) & =\left\langle\mathbf{1}_{ \pm}, \boldsymbol{M}_{0}(X, \cdot)\right\rangle,
\end{aligned}
$$

where $\phi_{T}+m_{X}=0$.

4.3. Long-time behavior. The final theorem we present provides us an asymptotic formula for the behavior of the depth-averaged density as $t \rightarrow \infty$.

Theorem 4.3. If the initial conditions are $\boldsymbol{N}(x, y, 0)=\boldsymbol{N}_{0}(x, y)$, where $\boldsymbol{N}_{0}(x, y) \in$ $\boldsymbol{B}(\mathrm{D})$ and

$$
\widehat{\boldsymbol{N}}_{0}(k, y)=\sum_{n=-\infty}^{\infty} \sum_{j=1}^{4} \widehat{g}_{j, n}(k) \boldsymbol{v}_{j, n}(k, y)
$$

in $L^{2}([0,1])$ (for each $k$ ) for some coefficients $\widehat{g}_{j, n}$, then the density for the Broadwell model has the following asymptotic behavior:

$$
\rho(x, t)=\sqrt{\frac{\pi}{D t}} \exp \left[\frac{-x^{2}}{4 D t}\right]\left(\sum_{m=-\infty}^{\infty} c_{m}\left(e^{i \pi m(x-t)}+e^{i \pi m(x+t)}\right)+o\left(t^{-\frac{1}{2}}\right)\right),
$$

where $D=(2-\alpha) /(2 \alpha)$ and

$$
\begin{aligned}
c_{2 m} & =\frac{1}{4}\left\langle\widehat{\boldsymbol{N}}_{0}(2 m \pi, \cdot), \boldsymbol{v}_{1, m}(2 m \pi, \cdot)\right\rangle \\
c_{2 m+1} & =\frac{1}{4}\left\langle\widehat{\boldsymbol{N}}_{0}((2 m+1) \pi, \cdot), \boldsymbol{v}_{2, m}(2 m+1) \pi, \cdot\right\rangle .
\end{aligned}
$$

Expressions for the vectors $\boldsymbol{v}_{j, n}$ are derived in Section 4.4.1. Furthermore, if $\boldsymbol{N}_{0}=$ $\frac{1}{4} f(x) \mathbf{1}$ then $\rho(x, 0)=f(x)$ and it follows from the above expressions that

$$
\rho(x, t)=\sqrt{\frac{\pi}{16 D t}} \exp \left[\frac{-x^{2}}{4 D t}\right]\left(\sum_{m=-\infty}^{\infty} \widehat{f}(\pi m)\left(e^{i \pi m(x-t)}+e^{i \pi m(x+t)}\right)+o\left(t^{-\frac{1}{2}}\right)\right) .
$$

Remark. If we knew that the eigenfunctions $\left\{\boldsymbol{v}_{j, n}(k, y)\right\}$ were, for all $k$, complete in $L^{2}[0,1]$ we could remove the condition given by (4.14).

Theorem 4.3 indicates that for a class of initial conditions the long-time behavior is composed of oscillatory functions that have the fundamental solution of the diffusion equation as an envelope. In particular, this demonstrates that the convergence to the diffusion equation as described in Theorem 4.1 can not be better than weak convergence. Indeed, Figure 3.2(b) clearly shows these oscillations. 
4.4. Proof of Theorems. We prove in this section the above theorems. In particular, we begin by computing the solution to the 4-velocity Broadwell model (2.1)-(2.4).

4.4.1. Eigenfunction expansion. The proof of each of the above theorems relies on an eigenfunction expansion of the the Broadwell model, which we will now outline. We begin by Fourier transforming the Broadwell model in $x$ and assuming a solution of the form:

$$
\widehat{N}_{j}(k, y, t)=\widehat{n}_{j}(k, y) e^{-i \omega(k) t} \quad \text { for } \quad j=1,2,3,4 .
$$

Plugging this into the Fourier transformed Broadwell model results in a first order ODE in $y$ for each $\widehat{n}$; these are easily solved and in view of the boundary conditions we write the solutions as

$$
\begin{aligned}
& \widehat{n}_{1}=a_{1} \exp [i(\omega-k) y] \\
& \widehat{n}_{2}=a_{2} \exp [i(\omega+k)(1-y)] \\
& \widehat{n}_{3}=a_{3} \exp [i(\omega+k) y] \\
& \widehat{n}_{4}=a_{4} \exp [i(\omega-k)(1-y)] .
\end{aligned}
$$

Applying the boundary conditions we find that

$$
M(z) \boldsymbol{a}=0,
$$

where $\boldsymbol{a}=\left(a_{1}, a_{2}, a_{3}, a_{4}\right)^{T}$ and

$$
M(z)=\left(\begin{array}{cccc}
-2 & \alpha z p & 0 & (2-\alpha) z / p \\
\alpha z / p & -2 & (2-\alpha) z p & 0 \\
0 & (2-\alpha) z p & -2 & \alpha z / p \\
(2-\alpha) z / p & 0 & \alpha z p & -2
\end{array}\right)
$$

with $z=e^{i \omega}$ and $p=e^{i k}$. For nontrivial solutions we must find the values of $z$ for which the determinant vanishes. We will also need to compute the eigenvectors, $\boldsymbol{a}$, that correspond to these values of $z$. The determinant of $M(z)$ vanishes whenever

$$
P_{+}(z) P_{-}(z)=0 \quad \text { where } \quad P_{ \pm}(z)=1+(1-\alpha) z^{2} \pm \frac{(2-\alpha)}{2}\left(\frac{1}{p}+p\right) z .
$$

We define $z_{1}$ and $z_{2}$ such that $P_{-}\left(z_{1}\right)=0$ and $P_{-}\left(z_{2}\right)=0$. It follows that $z_{3}=-z_{1}$ and $z_{4}=-z_{2}$ satisfy $P_{+}\left(z_{3}\right)=0$ and $P_{+}\left(z_{4}\right)=0$. We can obtain an expression for $z_{1}$ entirely in terms of $k$ and $\alpha$ by solving $P_{-}(z)=0$ and substituting $p=e^{i k}$ :

$$
z_{1}(k)=\left((2-\alpha) \cos (k)-\sqrt{(2-\alpha)^{2} \cos ^{2}(k)-4(1-\alpha)}\right) /(2(1-\alpha)) .
$$

Furthermore, it follows easily that

$$
z_{2}(k)=-z_{1}(k+\pi), \quad z_{3}(k)=-z_{1}(k), \quad z_{4}(k)=z_{1}(k+\pi) .
$$

Therefore it is enough to understand $z_{1}(k)$. We note the following properties:

$$
\begin{aligned}
z_{1}(k) & =z_{1}(k+2 n \pi) \quad \text { where } \quad n= \pm 1, \pm 2, \ldots \ldots \\
z_{1}(k+2 n \pi) & =1+\frac{2-\alpha}{2 \alpha} k^{2}+O\left(k^{4}\right) .
\end{aligned}
$$


To compute the dispersion relationship we need to find $\omega_{j}$. Since $z_{1}=e^{i \omega_{1}}$,

$$
\begin{aligned}
\omega_{1}(k) & =-i \log \left(z_{1}\right) \\
& =i \Omega(k)+\theta(k)+2 \pi n, \quad n=0, \pm 1, \pm 2, \ldots,
\end{aligned}
$$

where $\Omega(k)=-\log \left|z_{1}\right|$ and $\theta(k)=\cos ^{-1}\left(\operatorname{Re}\left(z_{1}\right) /\left|z_{1}\right|\right)$. Since $\omega_{1}$ is a $2 \pi$-periodic function of $k$, it is enough to understand the behavior of $\omega_{1}$ for $0 \leq k \leq 2 \pi$. It easy to see that

$$
z_{1}=\left\{\begin{array}{cc}
\text { complex } \\
\text { real } & \text { if } k_{c}<k<\pi-k_{c} \text { or } \pi+k_{c}<k<2 \pi-k_{c} \\
\text { otherwise }
\end{array}\right.
$$

where

$$
k_{c}=\cos ^{-1}\left(\frac{2 \sqrt{1-\alpha}}{2-\alpha}\right) .
$$

We observe that if $\operatorname{Im}\left(z_{1}\right)=0$ then

$$
\theta=\left\{\begin{array}{ccc}
0 & \text { if } & \operatorname{Re}(z)>0 \\
\pm \pi & \text { if } & \operatorname{Re}(z)<0
\end{array}\right.
$$

where the sign is chosen so that $\theta$ is continuous. On the other hand, we note if $\operatorname{Im}\left(z_{1}\right) \neq$ 0 then one can easily check that $\left|z_{1}\right|=(1-\alpha)^{-1 / 2}$ and $\cos (\theta)=\cos (k) / \cos \left(k_{c}\right)$. We also observe that if we expand $\Omega$, then we find that

$$
\Omega(k)=-\frac{2-\alpha}{2 \alpha} k^{2}+O\left(k^{4}\right) .
$$

We collect the above results and summarize them in Figure 4.1. In view of the above discussion and equation (4.18), we have the eigenfrequencies:

$$
\begin{aligned}
& \omega_{1, n}(k)=i \Omega(k)+\theta(k)+2 n \pi \\
& \omega_{2, n}(k)=i \Omega(k+\pi)+\theta(k+\pi)+(2 n+1) \pi \\
& \omega_{3, n}(k)=i \Omega(k)+\theta(k)+(2 n+1) \pi \\
& \omega_{4, n}(k)=i \Omega(k+\pi)+\theta(k+\pi)+2 n \pi .
\end{aligned}
$$

The eigenvectors corresponding the $z_{j}$ s described above are

$$
\boldsymbol{a}_{1}=\left(\begin{array}{c}
1 \\
E_{1} \\
E_{1} \\
1
\end{array}\right), \quad \boldsymbol{a}_{2}=\left(\begin{array}{c}
1 \\
E_{2} \\
E_{2} \\
1
\end{array}\right), \quad \boldsymbol{a}_{3}=\left(\begin{array}{c}
1 \\
-E_{1} \\
E_{1} \\
-1
\end{array}\right), \quad \text { and } \quad \boldsymbol{a}_{4}=\left(\begin{array}{c}
1 \\
-E_{2} \\
E_{2} \\
-1
\end{array}\right),
$$

where

$$
E_{j}=\frac{\left(2 p-(2-\alpha) z_{j}\right)}{\alpha z_{j} p^{2}} \quad \text { for } j=1,2
$$

We also note the following:

$$
E_{1}(k=2 m \pi)=1 \quad \text { and } \quad E_{1}(k=(2 m+1) \pi)=-1
$$



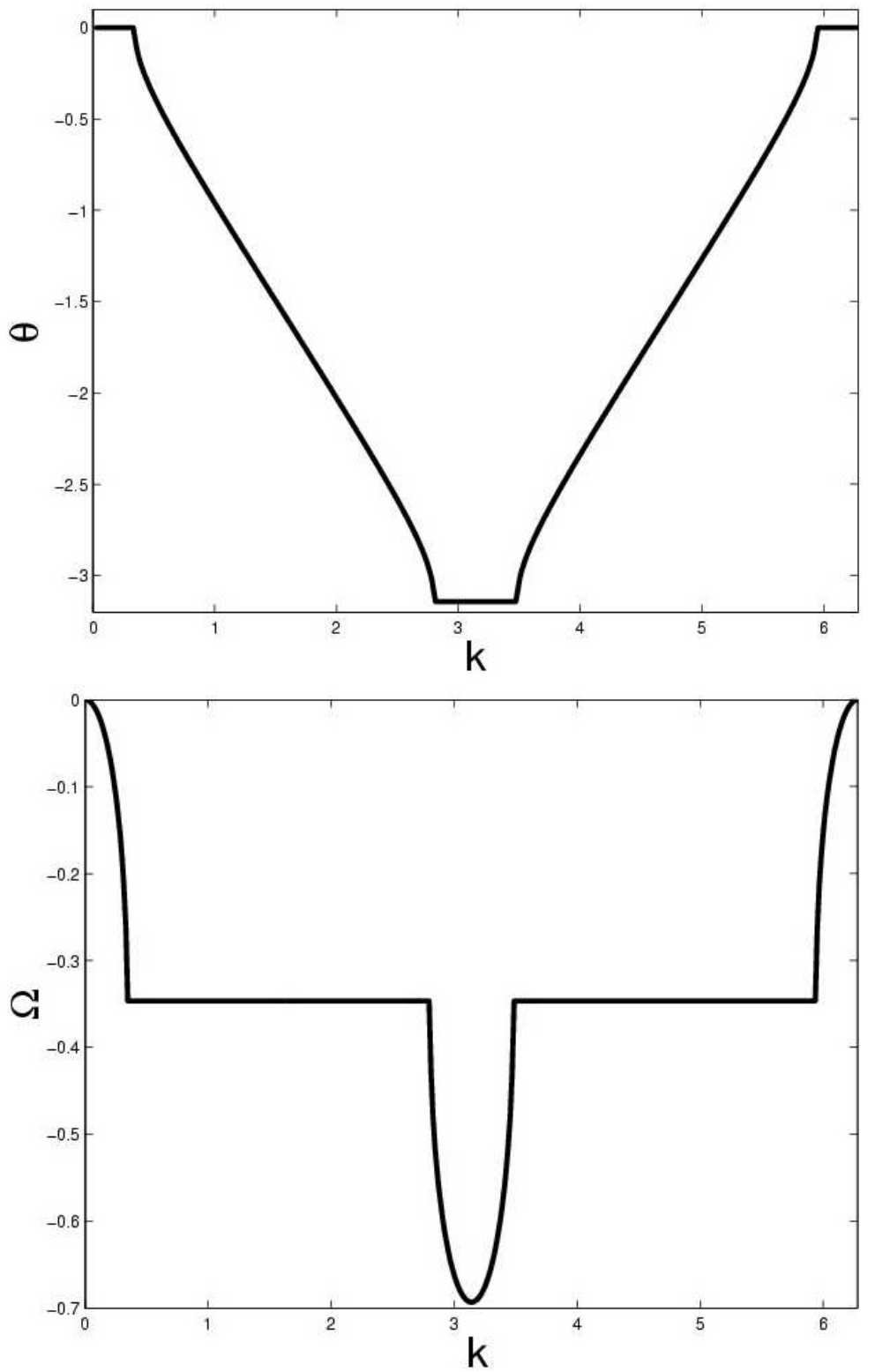

FIG. 4.1. A plot of $\theta$ and $\Omega$ as functions of $k$ for $\alpha=\frac{1}{2}$. The point on the $k$ axis where $\theta$ first becomes nonzero (moving away from the origin) marks $k_{c}$.

$$
E_{2}(k=2 m \pi)=-1 \quad \text { and } \quad E_{2}(k=(2 m+1) \pi)=1 .
$$

Equations (4.21) and (4.22) are valid if $\alpha$ is strictly bounded away from zero. On the other hand,

$$
\lim _{\varepsilon \rightarrow 0} E_{1}(k=w \varepsilon, \alpha=2 \Gamma \varepsilon)=\frac{\Gamma}{-i w+\sqrt{\Gamma^{2}-w^{2}}} \equiv F_{1}(w, \Gamma)
$$




$$
\lim _{\varepsilon \rightarrow 0} E_{2}(k=w \varepsilon, \alpha=2 \Gamma \varepsilon)=\frac{\Gamma}{-i w-\sqrt{\Gamma^{2}-w^{2}}} \equiv F_{2}(w, \Gamma) .
$$

Combining the results for the eigenvectors and eigenfrequencies we obtain the following eigenfunctions:

$$
\boldsymbol{v}_{j, n}(k, y)=\left(\begin{array}{l}
a_{j, 1} \exp \left[i\left(\omega_{j, n}-k\right) y\right] \\
a_{j, 2} \exp \left[i\left(\omega_{j, n}+k\right)(1-y)\right] \\
a_{j, 3} \exp \left[i\left(\omega_{j, n}+k\right) y\right] \\
a_{j, 4} \exp \left[i\left(\omega_{j, n}-k\right)(1-y)\right]
\end{array}\right) .
$$

4.5. Orthogonality results. Unfortunately the set of eigenfunctions given by (4.25) is not an orthogonal set; however, these eigenfunctions have some orthogonality properties that turn out to be sufficient for our needs. We will outline these properties in this section. If we define the inner product as in (4.1), then one can verify by a direct computation using equations (4.21) and (4.22) that

$$
\left\langle\boldsymbol{v}_{1, n}(2 m \pi, \cdot), \boldsymbol{v}_{j, \ell}(2 m \pi, \cdot)\right\rangle=4 \delta_{1 j} \delta_{n \ell}
$$

and

$$
\left\langle\boldsymbol{v}_{2, n}((2 m+1) \pi, \cdot), \boldsymbol{v}_{j, \ell}((2 m+1) \pi, \cdot)\right\rangle=4 \delta_{2 j} \delta_{n \ell} .
$$

One can also establish, recalling that $\mathbf{1}=(1,1,1,1)^{T}$, by direct computation that

$$
\left\langle\mathbf{1}, v_{1, n}(2 m \pi, \cdot)\right\rangle= \begin{cases}4 & \text { if } n=m=0 \\ 2 & \text { if } n= \pm m \\ 0 & \text { otherwise }\end{cases}
$$

and

$$
\begin{gathered}
\left\langle\mathbf{1}, v_{2, n}((2 m+1) \pi, \cdot)\right\rangle= \begin{cases}2 & \text { if } 2 n+1= \pm(2 m+1) \\
0 & \text { otherwise }\end{cases} \\
\left\langle\mathbf{1}, \boldsymbol{v}_{3, n}(k, \cdot)\right\rangle=\left\langle\mathbf{1}, \boldsymbol{v}_{4, n}(k, \cdot)\right\rangle=0 .
\end{gathered}
$$

In expressions $(4.26)-(4.30), j=1,2,3,4$ and $\ell, n, m=0, \pm 1, \pm 2, \pm 3, \ldots$.

Proposition 4.1. The set of vectors $\left\{\left\{\boldsymbol{v}_{j, n}(0, y)\right\}_{j=1}^{4}\right\}_{n=-\infty}^{\infty}$ is complete in $L^{2}[0,1]$.

Proof. Define the following $4 \times 4$ matrices:

$$
W_{j}= \begin{cases}\operatorname{diag}(1,1,1,1) & \text { if } j=1,3 \\ \operatorname{diag}\left(e^{2 \Omega(\pi) y}, e^{2 \Omega(\pi)(1-y)}, e^{2 \Omega(\pi) y}, e^{2 \Omega(\pi)(1-y))}\right) & \text { if } j=2,4 .\end{cases}
$$

One can use $W_{j}$ to formulate the following orthogonality condition:

$$
\left\langle\boldsymbol{v}_{j, n}(0, \cdot), W_{\ell} \boldsymbol{v}_{\ell, m}(0, \cdot)\right\rangle=4 \delta_{j, \ell} \delta_{m, n}
$$

An eigenfunction expansion for $\boldsymbol{f}(y)$ can be deduced using (4.32), and we find that

$$
\boldsymbol{f}_{N}(y)=\frac{1}{4} \sum_{n=-N}^{N} \sum_{j=1}^{4}\left\langle\boldsymbol{v}_{j, n}(0, \cdot), W_{j} \boldsymbol{f}(\cdot)\right\rangle \boldsymbol{v}_{j, n}(0, y)
$$

In section 4.6.4 (Lemma 4.1), we prove for any $\boldsymbol{f} \in L^{2}[0,1]$ that

$$
\lim _{N \rightarrow \infty}\left\|\boldsymbol{f}-\boldsymbol{f}_{N}\right\|_{2}=0 \text {. }
$$

This completes the proof of Proposition 4.1. 
4.6. Fourier Transform. Returning to (4.15) we see that the Fourier transform of $\boldsymbol{N}=\left(N_{1}, N_{2}, N_{3}, N_{4}\right)^{T}$ is

$$
\widehat{\boldsymbol{N}}=\sum_{n=-\infty}^{\infty} \sum_{j=1}^{4} \widehat{g}_{j, n}(k) \boldsymbol{v}_{j, n}(k, y) e^{-i \omega_{j, n}(k) t}
$$

where $\widehat{g}_{j, n}(k)$ comes from expanding the initial condition:

$$
\widehat{\boldsymbol{N}}_{0}(k, y)=\sum_{n=-\infty}^{\infty} \sum_{j=1}^{4} \widehat{g}_{j, n}(k) \boldsymbol{v}_{j, n}(k, y) .
$$

Since

$$
\rho(x, t)=\langle\mathbf{1}, \boldsymbol{N}\rangle \quad \text { and } \quad \widehat{\rho}(k, t)=\langle\mathbf{1}, \widehat{\boldsymbol{N}}\rangle,
$$

we can use equation (4.30) to write:

$$
\widehat{\rho}(k, t)=\widehat{\rho}_{1}(k, t)+\widehat{\rho}_{2}(k, t),
$$

where

$$
\widehat{\rho}_{j}(k, t)=\sum_{n=-\infty}^{\infty} G_{j, n}(k) e^{-i \omega_{j, n}(k) t}
$$

and

$$
G_{j, n}(k)=\widehat{g}_{j, n}(k)\left\langle\mathbf{1}, \boldsymbol{v}_{j, n}(k, y)\right\rangle .
$$

We will use the results of this section to prove the three theorems.

4.6.1. Proof of theorem 4.1. We start by taking the inverse Fourier transform of $\hat{\rho}_{j}$. It is convenient to break up the integral in intervals of length $2 \pi$ due to the periodic nature of $\omega$ as outlined in the section above. We have that

$$
\begin{aligned}
\rho_{j}(x, t) & =\int_{-\infty}^{\infty} \widehat{\rho}_{j}(k, t) e^{i k x} d k \\
& =\sum_{m=-\infty}^{\infty} \int_{\pi(2 m-1)}^{\pi(2 m+1)} \widehat{\rho}_{j}(k, t) e^{i k x} d k \\
& =\sum_{m=-\infty}^{\infty} \sum_{n=-\infty}^{\infty} A_{n, m}^{(j)} e^{-i \pi 2 n t},
\end{aligned}
$$

where

$$
A_{n, m}^{(j)}=\int_{(2 m-1) \pi}^{(2 m+1) \pi} G_{j, n} e^{-i \omega_{j, n}(k) t} e^{i k x} d k .
$$

Let $k=2 \pi m+w$ and we can write

$$
A_{n, m}^{(j)}=e^{i 2 m \pi x} I_{n, m}^{(j)},
$$


where

$$
I_{n, m}^{(j)}=\int_{-\pi}^{\pi} G_{j, n}(2 m \pi+w) e^{-i \omega_{j, 0} t} e^{i w x} d w .
$$

In expression (4.42) we have made use of he periodicity of $\omega$. Therefore we can write

$$
\rho_{j}(x, t)=\sum_{m=-\infty}^{\infty} \sum_{n=-\infty}^{\infty} I_{n, m}^{(j)} e^{-i 2 \pi(m x-n t)} .
$$

Under the assumptions of the Theorem, the initial conditions for $\boldsymbol{N}$ take the form $\boldsymbol{N}(x, y, 0)=\boldsymbol{M}_{0}(x / \lambda, y) / \lambda$. Since $\boldsymbol{M}_{0} \in \boldsymbol{B}(\mathrm{D})$ then $\widehat{\boldsymbol{M}}_{0}(\cdot, y) \in L^{1}(\mathrm{R})$. It also follows from our rescaling that

$$
\widehat{g}_{j, n}(k) \rightarrow \widehat{g}_{j, n}(\lambda k)
$$

Therefore we can write $I_{n, m}^{(j)}$ as

$$
I_{n, m}^{(j)}=\int_{-\pi}^{\pi} G_{j, n}(2 m \pi+w) e^{-i \omega_{j, 0}(w) t} e^{i w x} d w
$$

where

$$
G_{j, n}(2 m \pi+w)=\widehat{g}_{j, n}(2 \pi m \lambda+\lambda w)\left\langle\mathbf{1}, \boldsymbol{v}_{j, n}(2 m \pi+w)\right\rangle .
$$

We now use the rescaled variables to compute:

$$
\psi_{\lambda}(X, T)=\psi_{1}(X, T)+\psi_{2}(X, T)
$$

where

$$
\psi_{j}(X, T)=\sum_{m=-\infty}^{\infty} \sum_{n=-\infty}^{\infty} J_{n, m}^{(j)} e^{-i 2 \pi \lambda(m X-n T \lambda)}
$$

with

$$
J_{n, m}^{(j)}=\int_{-\lambda \pi}^{\lambda \pi} \widehat{g}_{j, n}(2 \pi m \lambda+k)\left\langle\mathbf{1}, \boldsymbol{v}_{1, n}\left(2 m \pi+\frac{k}{\lambda}\right)\right\rangle e^{-i \omega_{j, 0}\left(\frac{k}{\lambda}\right) \lambda^{2} T} e^{i k X} d k .
$$

One can show using the results of Section 4.4.1 that

$$
\lim _{\lambda \rightarrow \infty} \omega_{1,0}\left(\frac{k}{\lambda}\right) \lambda^{2}=-i D k^{2}
$$

and

$$
\lim _{\lambda \rightarrow \infty} \omega_{2,0}\left(\frac{k}{\lambda}\right) \lambda^{2}=-i \infty
$$

Clearly from (4.49) we find $\lim _{\lambda \rightarrow \infty} J_{n, m}^{(2)}=0$. We will examine the behavior of $J_{n, m}^{(1)}$ as $\lambda \rightarrow \infty$. First we consider the case $n=m=0$ :

$$
J_{0,0}^{(1)}=\int_{-\lambda \pi}^{\lambda \pi} \widehat{g}_{0,0}(k)\left\langle\mathbf{1}, \boldsymbol{v}_{1,0}\left(\frac{k}{\lambda}\right)\right\rangle e^{-i \omega_{1,0}\left(\frac{k}{\lambda}\right) \lambda^{2} T} e^{i k X} d k .
$$


By our hypothesis on the initial conditions $g_{0,0}(k) \in L^{1}$, and we can use the dominated convergence theorem to verify that

$$
\lim _{\lambda \rightarrow \infty} J_{0,0}^{(1)}=\int_{-\infty}^{\infty} 4 \widehat{g}_{1,0}(k) e^{i k X} e^{-D k^{2} T} d k .
$$

Turning to the case $m \neq 0$ and $n \neq 0$; since $\widehat{g}_{j, n}(k) \in L^{1}$ it follows that

$$
J_{n, m}^{(j)}<\infty .
$$

Using (4.50), (4.51), and recalling that $\lim _{\lambda \rightarrow \infty} J_{n, m}^{(2)}=0$, it follows from (4.46) and (4.47) that

$$
\psi_{\lambda}(X, T) \rightarrow \psi(X, T) \quad \text { weakly as } \lambda \rightarrow \infty,
$$

where

$$
\psi(X, T)=\int_{-\infty}^{\infty} 4 \widehat{g}_{1,0}(k) e^{i k X-k^{2} D T} d k,
$$

which is a solution of the diffusion equation. To complete our proof we must compute $\widehat{g}_{1,0}$ in terms the initial conditions. Combining (4.36) and (4.44) we have that

$$
\widehat{\boldsymbol{M}}_{0}(\lambda k, y)=\sum_{j=1}^{4} \sum_{m=-\infty}^{\infty} \widehat{g}_{j, m}(\lambda k) \boldsymbol{v}_{j, m}(k, y) .
$$

We let $w=\lambda k$ and take $\lambda \rightarrow \infty$ to obtain that

$$
\widehat{\boldsymbol{M}}_{0}(w, y)=\sum_{j=1}^{4} \sum_{m=-\infty}^{\infty} \widehat{g}_{j, m}(w) \boldsymbol{v}_{j, m}(0, y) .
$$

Our choice of initial conditions implies that $\widehat{\boldsymbol{M}}_{0}(\cdot, y) \in L^{2}[0,1]$. By Proposition 4.1 the eigenfunctions, $\left\{\boldsymbol{v}_{j, m}(0, y)\right\}$, are complete in $L^{2}[0,1]$. This combined with (4.32) indicates that we can uniquely determine $\widehat{g}_{j, m}$ for any $\widehat{\boldsymbol{M}}_{0}(\cdot, y) \in L^{2}[0,1]$.

To finish, we use (4.26) to obtain that

$$
\widehat{g}_{1,0}(w)=\frac{1}{4}\left\langle\boldsymbol{v}_{1,0}(0, \cdot), \widehat{\boldsymbol{M}}_{0}(w, \cdot)\right\rangle=\frac{1}{4}\left\langle\mathbf{1}, \widehat{\boldsymbol{M}}_{0}(w, \cdot)\right\rangle,
$$

and the proof of Theorem 4.1 is complete.

4.6.2. Proof of Theorem 4.2. In this theorem the initial conditions are the same as for Theorem 4.1, and the expression for $I_{n, m}^{(j)}$ is thereby the same:

$$
I_{n, m}^{(j)}=\int_{-\pi}^{\pi} G_{j, n}(2 m \pi+w) e^{-i \omega_{j, 0}(w, \alpha) t} e^{i w x} d w,
$$

where $G_{j, n}(2 m \pi+w)$ is given by (4.45) and we have written $\omega_{j, 0}$ so that its dependence on $\alpha$ is explicitly recognized. We now use the rescaled variables (4.8) to compute:

$$
\phi_{\lambda}(X, T)=\phi_{1}(X, T)+\phi_{2}(X, T),
$$


where

$$
\phi_{j}(X, T)=\sum_{m=-\infty}^{\infty} \sum_{n=-\infty}^{\infty} J_{n, m}^{(j)} e^{-i 2 \pi \lambda(m X-n T)}
$$

with

$$
J_{n, m}^{(j)}=\int_{-\lambda \pi}^{\lambda \pi} \widehat{g}_{j, n}(2 m \pi \lambda+k)\left\langle\mathbf{1}, \boldsymbol{v}_{j, n}\left(2 m \pi+\frac{k}{\lambda}, \frac{2 \Gamma}{\lambda}, y\right)\right\rangle e^{-i \lambda \omega_{j, 0}\left(\frac{k}{\lambda}, \frac{2 \Gamma}{\lambda}\right) T} e^{i k X} d k
$$

and $\boldsymbol{v}_{j, n}=\boldsymbol{v}_{j, n}(k, \alpha, y)$.

By expanding $\lambda \omega_{j, 0}\left(\frac{k}{\lambda}, \frac{2 \Gamma}{\lambda}\right)$ in series in $\lambda^{-1}$ one can prove that

$$
\lim _{\lambda \rightarrow \infty} \lambda \omega_{j, 0}\left(\frac{k}{\lambda}, \frac{2 \Gamma}{\lambda}\right)=\sigma_{j}(k),
$$

where $\sigma_{j}(k)$ is given by (4.11). In addition one can show that

$$
\lim _{\lambda \rightarrow \infty} \boldsymbol{v}_{j, n}\left(2 \pi m+\frac{k}{\lambda}, \frac{2 \Gamma}{\lambda}, y\right)=\boldsymbol{u}_{j, n}(m, y),
$$

with

$$
\boldsymbol{u}_{j, n}(m, y)=\left(\begin{array}{c}
\exp [2 \pi i(n-m) y] \\
F_{j} \exp [2 \pi i(n+m)(1-y)] \\
F_{j} \exp \left[2 \pi i\left(n+m+\frac{1}{2}\right) y\right] \\
\left.\exp \left[2 \pi i\left(n-m+\frac{1}{2}\right)(1-y)\right)\right]
\end{array}\right)
$$

where $j=1,2$ and $F_{j}$ are given by (4.23) and (4.24). The expressions for $j=3,4$ follow in a straightforward way. One can prove the following orthogonality relationship about these vectors:

$$
\begin{array}{lrl}
\left\langle\overline{\boldsymbol{u}}_{j, n}(0, \cdot), \boldsymbol{u}_{j, m}(0, \cdot)\right\rangle & =2\left(1+F_{j}^{2}\right) \delta_{n,-m} & \text { for } \quad j=1,2 \\
\left\langle\overline{\boldsymbol{u}}_{j, n}(0, \cdot), \boldsymbol{u}_{j, m}(0, \cdot)\right\rangle & =2\left(1+F_{j-2}^{2}\right) \delta_{n,-(m+1)} & \text { for } \quad j=3,4 \\
\left\langle\overline{\boldsymbol{u}}_{i, n}(0, \cdot), \boldsymbol{u}_{j, m}(0, \cdot)\right\rangle=0 & \text { if } \quad i \neq j .
\end{array}
$$

We can establish that $\boldsymbol{u}_{i, n}(0, y)$ can be used to form a complete basis in $L^{2}[0,1]$ using a similar argument as was given in Proposition 4.1. In addition we have the identity:

$$
\left\langle\mathbf{1}, \boldsymbol{u}_{j, 0}(0, \cdot)\right\rangle=2\left(1+F_{j}\right) .
$$

We proceed in a similar fashion as in Theorem 4.1, using (4.56), and prove that

$$
\lim _{\lambda \rightarrow \infty} J_{0,0}^{(j)}=\int_{-\infty}^{\infty} g_{j, 0}(k)\left(1+F_{j}(k, \Gamma)\right) e^{i\left(k x-\sigma_{j}(k)\right) t} d k,
$$

and if $m \neq 0$ and $n \neq 0$ then

$$
\lim _{\lambda \rightarrow \infty} J_{n, m}^{(j)}<\infty
$$

Therefore we have that

$$
\phi_{\lambda}(X, T) \rightarrow \phi(X, T) \quad \text { weakly as } \quad \lambda \rightarrow \infty
$$


where

$$
\phi(X, T)=\int_{-\infty}^{\infty}\left[\phi_{1}(k) e^{-i \sigma_{1}(k) T}+\phi_{2}(k) e^{-i \sigma_{2}(k) T}\right] e^{i k X} d k
$$

with $\phi_{j}(k)=\left(1+F_{j}(k, \Gamma)\right) \widehat{g}_{j, 0}(k)$.

To complete the proof we must compute $\phi_{j}(k)$ in terms of the initial conditions. Recall from (4.36) that $\widehat{g}_{j, n}(\lambda k)$ must satisfy:

$$
\widehat{\boldsymbol{M}}_{0}(\lambda k, y)=\sum_{j=1}^{4} \sum_{n=-\infty}^{\infty} \widehat{g}_{j, n}(\lambda k) \boldsymbol{v}_{j, n}(k, \alpha, y),
$$

which we write as

$$
\widehat{\boldsymbol{M}}_{0}(w, y)=\sum_{j=1}^{4} \sum_{n=-\infty}^{\infty} \widehat{g}_{j, n}(w) \boldsymbol{v}_{j, n}(w / \lambda, 2 \Gamma / \lambda, y) .
$$

Now let $\lambda \rightarrow \infty$ to obtain that

$$
\widehat{\boldsymbol{M}}_{0}(w, y)=\sum_{j=1}^{4} \sum_{n=-\infty}^{\infty} \widehat{g}_{j, n}(w) \boldsymbol{u}_{j, n}(0, y)
$$

Our choice of initial conditions implies that $\widehat{\boldsymbol{M}}_{0}(\cdot, y) \in L^{2}[0,1]$ and we know that $\left\{\boldsymbol{u}_{j, m}(0, y)\right\}$ forms a complete basis in $L^{2}[0,1]$. We can use the orthogonality relations (4.55) to uniquely determine $\widehat{g}_{j, n}$ for any $\widehat{\boldsymbol{M}}_{0}(\cdot, y) \in L^{2}[0,1]$.

It follows from (4.59) and (4.55) that

$$
\widehat{g}_{j, 0}(w)=\frac{\left\langle\overline{\boldsymbol{u}}_{j, 0}(0, \cdot), \widehat{\boldsymbol{M}}_{0}(\cdot)\right\rangle}{2\left(1+F_{j}^{2}(w, \Gamma)\right)} \quad \text { for } \quad j=1,2 .
$$

Finally we note that

$$
\boldsymbol{u}_{j, 0}(0)=\frac{1}{2}\left(1+F_{j}\right) \mathbf{1}+\frac{1}{2}\left(1-F_{j}\right) \mathbf{1}_{ \pm} \quad \text { for } \quad j=1,2 .
$$

A straightforward but somewhat lengthy calculation yields that

$$
\begin{gathered}
\phi_{1}(k)=\frac{\sigma_{2} \widehat{\rho}_{0}-k \widehat{m}_{0}}{\sigma_{2}-\sigma_{1}} \\
\phi_{2}(k)=\frac{\sigma_{1} \widehat{\rho}_{0}-k \widehat{m}_{0}}{\sigma_{1}-\sigma_{2}},
\end{gathered}
$$

where $\rho_{0}=\left\langle\mathbf{1}, \boldsymbol{M}_{0}(X, \cdot)\right\rangle, m_{0}=\left\langle\mathbf{1}_{ \pm}, \boldsymbol{M}_{0}(X, \cdot)\right\rangle$, and $\sigma_{j}$ is given by (4.11). If we compare with the solution of the telegraph equation given by (4.10), we see that the proof of this theorem is complete. 
4.6.3. Proof of Theorem 4.3. We again start by taking the inverse Fourier transform of $\widehat{\rho}_{1}$. We have that

$$
\begin{aligned}
\rho_{1}(x, t) & =\int_{-\infty}^{\infty} \widehat{\rho}_{1}(k, t) e^{i k x} d k \\
& =\sum_{m=-\infty}^{\infty} \int_{\pi(2 m-1)}^{\pi(2 m+1)} \widehat{\rho}_{1}(k, t) e^{i k x} d k \\
& =\sum_{m=-\infty}^{\infty} \sum_{n=-\infty}^{\infty} A_{n, m} e^{-i \pi 2 n t},
\end{aligned}
$$

where

$$
A_{n, m}=\int_{(2 m-1) \pi}^{(2 m+1) \pi} G_{1, n} e^{t(\Omega(k)-i \theta(k))} e^{i k x} d k .
$$

Let $k=2 \pi m+w$ and we can write:

$$
A_{n, m}=e^{i 2 m \pi x} I_{n, m}^{(1)}
$$

where

$$
I_{n, m}^{(1)}=\int_{-\pi}^{\pi} G_{1, n}(2 m \pi+w) e^{t(\Omega(w)-i \theta(w))} e^{i w x} d w .
$$

Here we have used the periodicity of $\Omega$ and $\theta$. Using this definition we can write:

$$
\rho_{1}(x, t)=\sum_{m=-\infty}^{\infty} \sum_{n=-\infty}^{\infty} I_{n, m}^{(1)} e^{-i 2 \pi(m x-n t)} .
$$

We can handle $\rho_{2}(x, t)$ is a similar fashion except the starting point is slightly different, namely

$$
\begin{aligned}
\rho_{2}(x, t) & =\int_{-\infty}^{\infty} \widehat{\rho}_{2}(k, t) e^{i k x} d k \\
& =\sum_{m=-\infty}^{\infty} \int_{2 m \pi}^{(2 m+2) \pi} \widehat{\rho}_{2}(k, t) e^{i k x} d k
\end{aligned}
$$

Following essentially the same steps we arrive at

$$
\begin{aligned}
\rho_{2}(x, t) & =\sum_{m=-\infty}^{\infty} \sum_{n=-\infty}^{\infty} I_{n, m}^{(2)} e^{-i \pi((2 m+1) x-(2 n+1) t)} \\
I_{n, m}^{(2)} & =\int_{-\pi}^{\pi} G_{2, n}((2 m+1) \pi+w) e^{t(\Omega(w)-i \theta(w))} e^{i w x} d w .
\end{aligned}
$$

Our choice of initial conditions guarantees that $\widehat{g}_{j, n}(k)$ will be bounded; consequently $G_{j, n}$ is also bounded and we can use Lemmas 4.2 and 4.3 to establish the following asymptotic expression:

$$
I_{n, m}^{(j)}=e^{-x^{2} / 4 D t}\left(\frac{\sqrt{\pi}}{\sqrt{D t}} G_{j, n}((2 m+j-1) \pi)+o\left(t^{-\frac{1}{2}}\right)\right) \quad \text { for } \quad j=1,2 .
$$


Next we use equations (4.38), (4.28) and (4.29) to show that

$$
\begin{aligned}
G_{1, n}(2 m \pi) & = \begin{cases}4 & \text { if } n=m=0 \\
2 & \text { if } n= \pm m \\
0 & \text { otherwise }\end{cases} \\
G_{2, n}((2 m+1) \pi) & = \begin{cases}2 & \text { if } 2 n+1= \pm(2 m+1) \\
0 & \text { otherwise. }\end{cases}
\end{aligned}
$$

Combining equations (4.66), (4.69), (4.71), (4.72), and (4.73) we have that

$$
\rho(x, t)=\frac{2 \sqrt{\pi}}{\sqrt{D t}} e^{-x^{2} / 4 D t}\left(\sum_{m=-\infty}^{\infty} c_{m}\left(e^{i \pi 2 m(x-t)}+e^{i \pi 2 m(x+t)}\right)+o\left(t^{-\frac{1}{2}}\right)\right)
$$

where

$$
c_{2 m}=\widehat{g}_{1, m}(2 m \pi) \quad \text { and } \quad c_{2 m+1}=\widehat{g}_{2, m}((2 m+1) \pi)
$$

To complete the proof of the theorem we must determine $c_{m}$ in terms of the initial conditions. Recall from (4.36) that

$$
\widehat{\boldsymbol{N}}_{0}(k, y)=\sum_{j=1}^{4} \sum_{m=-\infty}^{\infty} \widehat{g}_{j, m}(k) \boldsymbol{v}_{j, m}(k, y)
$$

We can use the orthogonality relations (4.26) and (4.27) to obtain that

$$
\widehat{g}_{1, m}(2 m \pi)=\frac{1}{4}\left\langle\widehat{\boldsymbol{N}}_{0}(2 m \pi, \cdot), \boldsymbol{v}_{1, m}(2 m \pi, \cdot)\right\rangle
$$

and

$$
\widehat{g}_{2, m}((2 m+1) \pi)=\frac{1}{4}\left\langle\widehat{\boldsymbol{N}}_{0}((2 m+1) \pi, \cdot), \boldsymbol{v}_{2, m}((2 m+1) \pi, \cdot)\right\rangle
$$

This completes the proof of Theorem 4.3.

\subsubsection{Three lemmas.}

Lemma 4.1. For any $\boldsymbol{f} \in L^{2}[0,1]$ its eigenfunction expansion as given by (4.33) converges in $L^{2}$. In other words, $\lim _{N \rightarrow \infty}\left\|\boldsymbol{f}-\boldsymbol{f}_{N}\right\|_{2}=0$.

Proof. The eigenfunction expansion given by (4.33) can be written as

$$
\boldsymbol{f}_{N}(y)=\int_{0}^{1} K(x, y) \boldsymbol{f}(y) d y
$$

where $K$ is the $4 \times 4$ matrix given by

$$
K(x, y)=\frac{1}{4} \sum_{n=-N}^{N} \sum_{j=1}^{4} \overline{\boldsymbol{v}}_{j, n}(0, x) \otimes W_{j} \boldsymbol{v}_{j, n}(0, y) .
$$


The entries of $K$ are given by

$$
\begin{aligned}
& \left.K_{11}=\frac{1}{4}\left(1+e^{\Omega(\pi)(y-x)}\right)\left(1+e^{i \pi(y-x)}\right)\right) D_{N}(x-y) \\
& K_{12}=\frac{1}{4}\left(1-e^{\Omega(\pi)(1-x-y)}\right)\left(1+e^{i \pi(x+y)}\right) D_{N}(x+y) \\
& K_{13}=\frac{1}{4}\left(1-e^{\Omega(\pi)(y-x))}\right)\left(1+e^{i \pi(x-y)}\right) D_{N}(x-y) \\
& K_{14}=\frac{1}{4}\left(1+e^{\Omega(\pi)(1-x-y)}\right)\left(1+e^{i \pi(x+y)}\right) D_{N}(x+y) \\
& K_{21}=\frac{1}{4}\left(1-e^{\Omega(\pi)(x+y-1)}\right)\left(1+e^{-i \pi(x+y)}\right) D_{N}(x+y) \\
& K_{22}=\frac{1}{4}\left(1+e^{\Omega(\pi)(x-y)}\right)\left(1+e^{i \pi(x-y)}\right) D_{N}(x-y) \\
& K_{23}=\frac{1}{4}\left(1+e^{\Omega(\pi)(x+y-1)}\right)\left(1+e^{-i \pi(x+y)}\right) D_{N}(x+y) \\
& K_{24}=\frac{1}{4}\left(1-e^{\Omega(\pi)(x-y)}\right)\left(1+e^{i \pi(y-x)}\right) D_{N}(x-y) \\
& K_{31}=\frac{1}{4}\left(1-e^{\Omega(\pi)(y-x)}\right)\left(1+e^{i \pi(x-y)}\right) D_{N}(x-y) \\
& K_{32}=\frac{1}{4}\left(1+e^{\Omega(\pi)(1-y-x)}\right)\left(1+e^{i \pi(x+y)}\right) D_{N}(x+y) \\
& K_{33}=\frac{1}{4}\left(1+e^{\Omega(\pi)(y-x)}\right)\left(1+e^{i \pi(x-y)}\right) D_{N}(x-y) \\
& K_{34}=\frac{1}{4}\left(1-e^{\Omega(\pi)(1-y-x)}\right)\left(1+e^{i \pi(x+y)}\right) D_{N}(x+y) \\
& K_{41}=\frac{1}{4}\left(1+e^{\Omega(\pi)(x+y-1)}\right)\left(1+e^{-i \pi(x+y)}\right) D_{N}(x+y) \\
& K_{42}=\frac{1}{4}\left(1-e^{\Omega(\pi)(x-y)}\right)\left(1+e^{i \pi(y-x)}\right) D_{N}(x-y) \\
& K_{43}=\frac{1}{4}\left(1-e^{\Omega(\pi)(x+y-1)}\right)\left(1+e^{-i \pi(y+x)}\right) D_{N}(x+y) \\
& K_{44}=\frac{1}{4}\left(1+e^{\Omega(\pi)(x-y)}\right)\left(1+e^{i \pi(y-x)}\right) D_{N}(x-y),
\end{aligned}
$$

where $D_{N}$ is the Dirichlet Kernel and is given by

$$
D_{N}(x)=\sum_{n=-N}^{N} e^{2 \pi n i x}=\frac{\sin ((2 N+1) \pi x)}{\sin (\pi x)}
$$

We now invoke the following theorem concerning the Dirichlet kernel (see for example $[32])$.

Theorem (Riesz). For $f \in L^{2}$

$$
\lim _{N \rightarrow \infty}\left\|f(x)-\int_{0}^{1} f(y) D_{N}(x-y) d y\right\|_{2}=0 .
$$

This theorem is valid in $L^{p}$ for $1<p<\infty$ but here we are only concerned with the $L^{2}$ case. In fact, we require a slight generalization of this theorem:

Let $g(x)$ be a $C^{\infty}$ function, then for $f \in L^{2}$

$$
\lim _{N \rightarrow \infty}\left\|f(x) g(0)-\int_{0}^{1} f(y) g(x-y) D_{N}(x-y) d y\right\|_{2}=0 .
$$

This modification follows directly from Riesz's Theorem. We can use (4.78) to show that

$$
\lim _{N \rightarrow \infty}\left\|f_{i}-\int_{0}^{1} K_{i i} f_{i} d y\right\|_{2}=0
$$


for $i=1,2,3,4$, and

$$
\lim _{N \rightarrow \infty}\left\|\int_{0}^{1} K_{i j} f_{j} d y\right\|_{2}=0
$$

for $(i, j)=(1,3),(3,1),(2,4),(4,2)$. To handle the other cases we observe that

$$
\begin{aligned}
\int_{0}^{1} f(x) g(x+y)(1+ & \left.e^{i \pi(x+y)}\right) D_{N}(x+y) d y \\
& =\int_{0}^{1} f(x) g(x-w+1)\left(1-e^{i \pi(x-w)}\right) D_{N}(x-w) d w .
\end{aligned}
$$

To derive this expression we have used the periodicity of the Dirichlet kernel $\left(D_{N}(x)=\right.$ $\left.D_{N}(x+1)\right)$. It then follows from (4.78) that

$$
\lim _{N \rightarrow \infty}\left\|\int_{0}^{1} f(x) g(x-w+1)\left(1-e^{i \pi(x-w)}\right) D_{N}(x-w) d w\right\|_{2}=0 .
$$

The proof of the lemma is complete.

Lemma 4.2. Consider

$$
g(x, t)=\int_{-\ell}^{\ell} e^{i k x} e^{\Omega(k) t} f(k) d k,
$$

where $f(k)$ is a bounded function and

$$
\begin{aligned}
& \Omega(k)+D k^{2} \leq 0 \\
& \Omega(k)+D k^{2}=o\left(k^{2}\right) .
\end{aligned}
$$

Then we have the following asymptotic expression:

$$
g(x, t)=e^{-x^{2} / 4 D t}\left(\frac{f(0) \sqrt{\pi}}{\sqrt{D t}}+o\left(t^{-\frac{1}{2}}\right)\right) .
$$

Proof. Let $k=w / \sqrt{t}$ and $s=x / \sqrt{t}$, then

$$
g(x, t)=e^{-s^{2} / 4 D} h(s, t) / \sqrt{t},
$$

where

$$
h(s, t)=\int_{-\infty}^{\infty} p(t, w, s) d w
$$

and

$$
p(t, w, s)=e^{s^{2} / 4 D} e^{i w s-D w^{2}} e^{t \Omega(w / \sqrt{t})+D w^{2}} f(w / \sqrt{t}) \chi_{[-\ell \sqrt{t}, \ell \sqrt{t}]} .
$$

In the above expression $\chi_{A}$ is the characteristic function of the set $A$. In view of hypothesis (4.81) we have that

$$
\lim _{t \rightarrow \infty} t \Omega(w / \sqrt{t})+D w^{2}=0
$$


Consequently

$$
\lim _{t \rightarrow \infty} p(t, w, s)=e^{i w s-D w^{2}} f(0) e^{s^{2} / 4 D} .
$$

We can use hypothesis (4.80) to obtain the following bound (uniform in $t$ ):

$$
|p(t, w, s)| \leq e^{-D w^{2}} e^{s^{2} / 4 D} \max _{[-\ell, \ell]} f(k) .
$$

We can now use the dominated convergence theorem to verify that

$$
\begin{gathered}
\lim _{t \rightarrow \infty} \int_{-\infty}^{\infty} p(t, w, s) d w=e^{s^{2} / 4 D} \int_{-\infty}^{\infty} e^{i w s-D w^{2}} f(0) d w \\
=\frac{f(0) \sqrt{\pi}}{\sqrt{D}} .
\end{gathered}
$$

Therefore we can conclude that

$$
h(s, t)=\frac{f(0) \sqrt{\pi}}{\sqrt{D}}+o(1) \quad \text { as } \quad t \rightarrow \infty .
$$

Combining (4.83),(4.86), and $s=x / \sqrt{t}$ completes the proof of Lemma 4.2.

Lemma 4.3. We have the following asymptotic expression for $I_{n, m}^{(j)}$ given by (4.65) and (4.70):

$$
I_{n, m}^{(j)}=e^{-x^{2} / 4 D t}\left(\frac{\sqrt{\pi}}{\sqrt{D t}} G_{j, n}((2 m+j-1) \pi)+o\left(t^{-\frac{1}{2}}\right)\right) \quad \text { for } \quad j=1,2 .
$$

Proof. We begin with the definition of $I_{n, m}^{(j)}$ :

$$
I_{n, m}^{(j)}=\int_{-\pi}^{\pi} G_{j, n}((2 m+j-1) \pi+w) e^{t(\Omega(w)-i \theta(w))} e^{i w x} d w .
$$

In view of the properties of $\Omega$ and $\theta$ outlined in Section 4.4.1, we can write the above equation as

$$
I_{n, m}^{(j)}=\int_{-k_{c}}^{k_{c}} G_{j, n}((2 m+j-1) \pi+w) e^{t \Omega(w)} e^{i w x} d w+O\left(e^{-t}\right) .
$$

One can expand the expression for $\Omega(w)$ in a power series in $w$ and verify that it satisfies the hypotheses of Lemma 4.2. By our hypothesis on the initial conditions $G_{j, n}$ is bounded. Hence we may apply Lemma 4.2 to the integral above and our proof is finished.

\section{Summary}

In this paper, we have examined the Broadwell model between two parallel plates with and without inter-particle collisions. In the collisionless case we present formal asymptotic arguments that indicate that the depth-averaged density should satisfy a telegraph equation. A similar analysis is performed to the Broadwell model with collisions. Here we find that the depth-averaged density should satisfy a telegraph equation coupled to an ODE which accounts for the effects of collisions. In addition, it is demonstrated that in both cases the predictions of our telegraph equations agree 
well with numerical calculations. In the collisionless case, we prove that under a diffusive scaling the depth-averaged density will converge weakly to a solution of a diffusion equation. We also prove that under a hyperbolic scaling the depth-averaged density will converge weakly to a solution of the telegraph equation. Finally, we derive an asymptotic formula for the long-time behavior of the depth-averaged density, which reveals oscillations indicating that the convergence discussed above must be weak.

Acknowledgments. PS thanks Paul Federbush and Jeff Rauch for helpful discussions. PS was supported, in part, by NSF grants DMS-0207420 and DMS 0244419. $\mathrm{AC}$ and JR were supported, in part, by the NSF VIGRE program.

\section{REFERENCES}

[1] K. Aoki and P. Degond, Homogenization of a flow in a periodic channel of small section, SIAM Multiscale Model. Simul., 1, 304-334, 2003.

[2] H. Babovsky, On Knudsen flows within thin tubes, J. Stat. Phys., 44, 865, 1986.

[3] H. Babovsky, Diffusion limit for flows in thin layers, SIAM J. Numer. Anal., 56, 1280, 1996.

[4] H. Babovsky, C. Bardos, and T. Platkowski, Diffusion approximation for a Knudsen gas in a thin domain with accommodation on the boundary, Asymptotic Anal., 3, 265-289, 1991.

[5] C. Bardos, F. Golse, and J-F. Colonna, Diffusion approximation and hyperbolic automorphisms of the torus, Physica D, 104, 32-60, 1997.

[6] C. Börgers, C. Greengard, and E. Thomann, The diffusion limit of free molecular-flow in thin plane Channels, SIAM J. Numer. Anal., 52, 1057, 1992.

[7] A. Bellouquid, From microscopic to macroscopic models: asymptotic analysis of the Broadwell model toward the wave equation, Math. Comput. Modelling, 36, 1169-1181, 2002.

[8] G.A. Bird, Molecular Gas Dynamics and the Direct Simulation of Gas Flows, Oxford University Press, New York, 1965.

[9] S. Boatto and F. Golse, Diffusion approximation of a Knudsen gas model: dependence of the diffusion constant upon the boundary condition, Asymptotic Anal., 31, 93-111, 2002.

[10] J.E. Broadwell, Shock structure in a simple discrete velocity gas, Phys. Fluids, 7, 1243, 1964.

[11] J.E. Broadwell, Study of rarefied shear flow by the discrete velocity method, J. Fluid Mech., 19, 401, 1964.

[12] R.E. Caflisch, Navier-Stokes and Boltzmann shock profiles for a model of gas dynamics, Comm. Pure Appl. Math., XXXII:521, 1979.

[13] R.E. Caflisch and G.C. Papanicolaou, The fluid-dynamical limit of a nonlinear model Boltzmann equation, Comm. Pure Appl. Math.,, XXXII:589, 1979.

[14] S. Chapman and T.G. Cowling, The Mathematical Theory of Non-Uniform Gases, Cambridge University Press, Cambridge, England, 1952.

[15] S.B. Choi, R.F. Barron, and R.O. Warrington, Fluid flow and heat transfer in microtubes, In Micromechanical Sensors, Actuators, and Systems, vol. 32, pp. 123. ASME, New York, 1991.

[16] P. Degond, V. Latocha, S. Mancini, and A. Mellet, Diffusion dynamics of an electron gas confined between two plates, Methods Appl. Anal., 9, 127-150, 2002.

[17] P. Degond and S. Mancini, Diffusion driven by collisions with the boundary, Asymp. Anal., 27, 47-73, 2001.

[18] C. Dogbé, Anomalous diffusion limit induced on a kinetic equation, J. Stat. Phys., 100, 603632, 2000.

[19] L.S. Fan, Y.C. Tai, and R.S. Muller, IC-processed electrostatic micro-motors, In Technical Digest, IEEE International Electron Devices Meeting, 666-669, San Francisco, CA, December 1988.

[20] M. Gad-el-hak, The fluid mechanics of microdevices - the Freeman scholar lecture, J. Fluids Eng., 121, 5, 1999.

[21] F. Golse, Anomalous diffusion limit for the Knudsen gas, Asymptotic Anal., 17, 1-12, 1998.

[22] C.-M. Ho and Y.-C. Tai, Micro-electro-mechanical systems (MEMS) and fluid flows, Ann. Rev. Fluid Mech., 30, 579, 1998.

[23] R.J. LeVeque, CLAWPACK software, available from http: //www. amath. washington. edu/ claw.

[24] R.J. LeVeque, Wave propagation algorithms for multi-dimensional hyperbolic systems, J. Comp. Phys., 131, 327-335, 1997. 
[25] R.J. LeVeque, Finite Volume Methods for Hyperbolic Problems, Cambridge University Press, 2002.

[26] T. Platkowski and R. Illner, Discrete velocity models of the Boltzmann equation: a survey on the mathematical aspects of the theory, SIAM Rev., 30, 213, 1988.

[27] J.C. Shih, C.-M. Ho, J.Q. Liu, and Y.-C. Tai, Monatomic and polyatomic gas flow through uniform microchannels, In ASME Int. Mech. Eng. Congress and Exposition, 59, 197-203, Nov. 1996.

[28] Y. Sone, Y. Waniguchi, and K. Aoki, One-way flow of a rarefied gas induced in a channel with a periodic temperature distribution, Phys. Fluids, 8, 2227, 1996.

[29] Y. Sone and M. Yoshimoto, Demonstration of a rarefied gas flow induced near the edge of a uniformly heated plate, Phys. Fluids, 9, 3530, 1997.

[30] D. Valougeorgis and S. Naris, Acceleration schemes of discrete velocity method: gaseous flows in rectangular microchannels, SIAM J. Sci. Comput., 25, 534, 2003.

[31] W.G. Vincenti and C.H. Kruger, Introduction to Physical Gas Dynamics, John Wiley \& Sons, New York, 1965.

[32] R.L. Wheeden and A. Zygmund, Measure and Integral - An Introduction to Real Analyis, Marcel Dekker, New York, 1977.

[33] P. Wu and W.A. Little, Measurement of the heat transfer characteristics of gas flow in a fine channel heat exchangers used for microminiature refrigerators, Cryogenics, 24, 415, 1984.

[34] P.Y. Wu and W.A. Little, Measurement of friction factors for the flow of gases in very fine channels used for microminiature Joule-Thomson refrigerators, Cryogenics, 23, 273, 1983.

[35] E. Zauderer, Partial Differential Equations of Applied Mathematics, John Wiley \& Sons, 2nd edition, 1998. 